\title{
Seasons, weather, and device-measured movement behaviors: a scoping review from 2006 to 2020
}

\author{
Taylor B. Turrisi', Kelsey M. Bittel', Ashley B. West', Sarah Hojjatinia², Sahar Hojjatinia ${ }^{3}$, Scherezade K. Mama4, \\ Constantino M. Lagoa ${ }^{3}$ and David E. Conroy ${ }^{1,5^{*}}$ (D)
}

\begin{abstract}
Background: This scoping review summarized research on (a) seasonal differences in physical activity and sedentary behavior, and (b) specific weather indices associated with those behaviors.

Methods: PubMed, CINAHL, and SPORTDiscus were searched to identify relevant studies. After identifying and screening 1459 articles, data were extracted from 110 articles with 118,189 participants from 30 countries (almost exclusively high-income countries) on five continents.

Results: Both physical activity volume and moderate-to-vigorous physical activity (MVPA) were greater in summer than winter. Sedentary behavior was greater in winter than either spring or summer, and insufficient evidence existed to draw conclusions about seasonal differences in light physical activity. Physical activity volume and MVPA duration were positively associated with both the photoperiod and temperature, and negatively associated with precipitation. Sedentary behavior was negatively associated with photoperiod and positively associated with precipitation. Insufficient evidence existed to draw conclusions about light physical activity and specific weather indices. Many weather indices have been neglected in this literature (e.g., air quality, barometric pressure, cloud coverage, humidity, snow, visibility, windchill).

Conclusions: The natural environment can influence health by facilitating or inhibiting physical activity. Behavioral interventions should be sensitive to potential weather impacts. Extreme weather conditions brought about by climate change may compromise health-enhancing physical activity in the short term and, over longer periods of time, stimulate human migration in search of more suitable environmental niches.
\end{abstract}

Keywords: Environment, Seasons, Meteorological concepts, Rain, Sunlight, Temperature, Wind, Exercise, Screen time

The global prevalence of insufficient physical activity is approximately $28 \%$ but exceeds $40 \%$ in some regions [1]. Physical activity promotion efforts have targeted determinants at multiple levels of the socio-ecological model, including the person (e.g., motivation), social environment

\footnotetext{
* Correspondence: conroy@psu.edu

'Department of Kinesiology, The Pennsylvania State University, University Park, PA 16802, USA

${ }^{5}$ Department of Preventive Medicine, Northwestern University, Chicago, IL, USA

Full list of author information is available at the end of the article
}

(e.g., family, peers), and built environment (e.g., access to equipment, neighborhood walkability) [2-4]. The natural environment includes a number of factors that influence physical activity, including seasons and weather. Weather is often cited as a perceived barrier to participation in movement behaviors [5-9]. Although weather conditions are not acutely modifiable, they are important to understand because of their ability to alter opportunities for physical activity and moderate the effectiveness of interventions targeting determinants at other levels. 
Two seminal reviews of research on seasonality, weather, and physical activity across the lifespan were published over a decade ago [10, 11]. Physical activity was typically greatest during spring and summer and lowest during winter, but regions with more extreme weather conditions sometimes yielded different conclusions. For example, a study conducted in Galveston, Texas, where the average temperature during summer months is over $28^{\circ} \mathrm{C}\left(82^{\circ} \mathrm{F}\right)$, revealed lower levels of physical activity in the summer than in winter [12]. However, the general pattern of seasonal trends demonstrated that people accumulated greater levels of physical activity in warmer and more arid conditions. Weather conditions were also found to have differing impacts on physical activity between sub-groups in the population $[10,13,14]$. For instance, physical activity was not associated with wind gusts for most individuals, but individuals with lower body mass were less active in the presence of stronger wind gusts than individuals with higher body mass [15].

The social context for research on weather and physical activity changed in three significant ways around the time of the seminal Tucker and Gilliland review [10]. First, public interest in weather and health increased following the 2007 Nobel Peace Prize that was awarded jointly to the Intergovernmental Panel on Climate Change and former US Vice President $\mathrm{Al}$ Gore [16]. As Earth's surface temperature rises, extreme weather conditions will increase air pollution and ultraviolet radiation exposure, increasing risk for cardiovascular and lung diseases as well as cancer [17]. Second, the mobile and wearable technology industries experienced major disruptions in 2007 due to the launch of the Apple iPhone and the founding of Fitbit $[18,19]$. The widespread adoption of mobile technologies such as smartphones and wearable activity monitors enabled researchers to monitor both physical activity and location-specific weather indices in real-time. Third, sedentary behavior - waking activity conducted in a seated or reclined posture involving low energy expenditure - has emerged as a distinct behavior that has important health consequences independent of physical activity levels $[20,21]$.

Despite summarizing over 60 studies with over 300, 000 participants from approximately 18 countries, these seminal reviews possess several limitations. First, although they both examined seasonal differences in physical activity, most findings at the time focused on a relatively narrow range of specific weather indices with temperature and precipitation being most common. Understanding associations between additional weather indices and physical activity could both explain seasonal differences and facilitate the development of just-in-time interventions that leverage information from short-term weather forecasts. Second, weather and physical activity data were aggregated into person-level summary measures, yet both weather and physical activity are dynamic. Understanding the timing of and changes in weather conditions is essential because current weather conditions are likely to have a more immediate influence on physical activity than average weather conditions. Third, both reviews focused on a general physical activity outcome. Physical activity can be quantified as total volume (to represent energy expended) or the duration of activities completed at specific intensities (to represent time allocated to different effort levels). Sedentary behavior may also be impacted as weather conditions alter people's activity choices. Additional reviews published since these seminal reviews have been limited by incomplete search strategies and a focus on narrow segments of the population [14, 22]. In light of these limitations and the broader context described earlier (increasing public interest in climate, advances in mobile technology, the emergence of sedentary behavior), an updated review of weather and movement behavior, including both physical activity and sedentary behavior, would be a valuable contribution.

A scoping review was conducted to examine associations between device-based measures of physical activity, sedentary behavior and a broad array of weather-related phenomena at different levels of specificity, ranging from seasons (e.g., spring, summer, fall, winter) to specific weather indices (e.g., humidity, precipitation, temperature). A scoping review was selected over a systematic review or meta-analysis based on the breadth of weather indices available and the need to both analyze the available evidence and identify existing knowledge gaps [23].

\section{Methods \\ Search strategy}

PubMed, the Cumulative Index of Nursing and Allied Health Literature (CINAHL), and SPORTDiscus electronic databases were searched from January 1, 2006 to October 31, 2020. This date range was chosen to capture all research since the end of the search period used by Tucker and Gilliland [10]. Three databases were selected based on their likelihood of including both environmental and health behavior data. Three main subject categories were included in our searches: movement-related behavior, movementrelated behavior measurement (i.e., intensity, volume), and weather. Movement-related behavior search terms related to physical activity or sedentary behavior were combined with "or" statements. These terms mirrored 
the search terms used to compile the literature for the 2018 Physical Activity Guidelines Advisory Committee [24]. Measurement search terms related to technologies commonly used to measure physical activity were combined with "or" statements (e.g., accelerometer or pedometer). Weather search terms related to seasonality or specific weather indices were combined with "or" statements (e.g., seasons or temperature or humidity or precipitation). The search strategies for each database used are available in Appendices 1,2 , and 3 . The searches were restricted to articles that (a) were written in English, (b) examined human subjects only, and (c) included empirical studies only (i.e., no review papers). This search strategy was constructed in consultation with a trained reference librarian and search specialist at The Pennsylvania State University. The review protocol was not preregistered.

\section{Selection process}

Articles were included if (a) physical activity or sedentary behavior was an outcome variable of interest, (b) physical activity data were collected using device-based measurements (e.g., accelerometer, pedometer), and (c) results involved associations or differences between either movement-related behavior and either seasonality or weather indices. Articles were excluded if (a) studies were not published in English, (b) samples included non-human subjects, (c) results were limited to prevalence rates or other descriptive data, or (d) physical activity data were collected using self-report measures exclusively.

Titles and abstracts were independently reviewed in a blinded manner by coders trained by the first author using the eligibility criteria described above. The first author exported citations for each article found in the PubMed, CINAHL, and SPORTDiscus searches, and uploaded these citations into Rayyan. Rayyan is a web application that is used to facilitate collaborative screening of titles and abstracts for reviews [25, 26]. Each coder accessed their assigned articles via the Rayyan web interface, reviewed titles and abstracts, and recorded decisions to include or exclude each article. Figure 1 summarizes study selection [27].

\section{Data extraction}

Articles containing studies that met inclusion criteria during full-text review were advanced for data extraction. Prior to data extraction, the first author and four trained coders used a standardized coding guide to code three identical papers that were eligible for data extraction. The coding guide was developed to ensure that sufficient descriptive information was collected to properly characterize associations of interest.
The coders then met to compare codes and discuss disagreements to promote consistency for final extraction. After this calibration exercise, each coder independently extracted data from the remaining articles using the standardized coding guide. Prior to analysis, the first author reviewed each article to ensure that the necessary data was extracted in a manner consistent with the coding guide.

A copy of the coding guide is available in supplementary online files. Extracted sample characteristics included age, sex, education, race, and the country where the data were collected. If countries included multiple diverse climate zones, the region of the country was also collected. Physical activity, sedentary behavior, weather indices (temperature, precipitation, wind speed, photoperiod, snow, cloud coverage, humidity, visibility, barometric pressure, windchill, and air quality; see Appendix 4 for definitions), and seasonality were all characterized by assessment timeframe and method of measurement. Research designs were classified as cross-sectional or longitudinal. The following statistics were extracted when available: t-scores, F-scores, correlation coefficients, $\beta$ coefficients, $p$-values, effect sizes, and odds ratios. Significance thresholds were kept consistent with the author's prespecified level of significance, and all results were coded having a positive or negative association, or failing to reach significance.

\section{Evidence grading}

All of the available evidence from independent samples was observational so all studies were deemed to have a high risk of bias. Instead of rating study-level bias, evidence was graded based on the quantity and consistency of findings when five of more studies were available for a specific comparison or association. A "strong" grade was assigned when a conclusion was based on highly consistent findings related to the direction of a difference or association (or moderately consistent findings when a large number of studies was available). A "moderate" grade was assigned when a conclusion was based on mixed findings but the preponderance of evidence pointed to a consistent direction of a difference or association. A "limited" grade was assigned when a conclusion could not be drawn because of equivocal evidence related to the direction of the difference or association. When fewer than five studies were available, we noted that a grade was not assignable. This grading system was drawn from criteria used by the 2018 Physical Activity Guidelines Advisory Committee and adapted to match the state of this literature [24].

\section{Results}

A total of 1459 unique articles were identified during the initial search. Following title and abstract screening, 


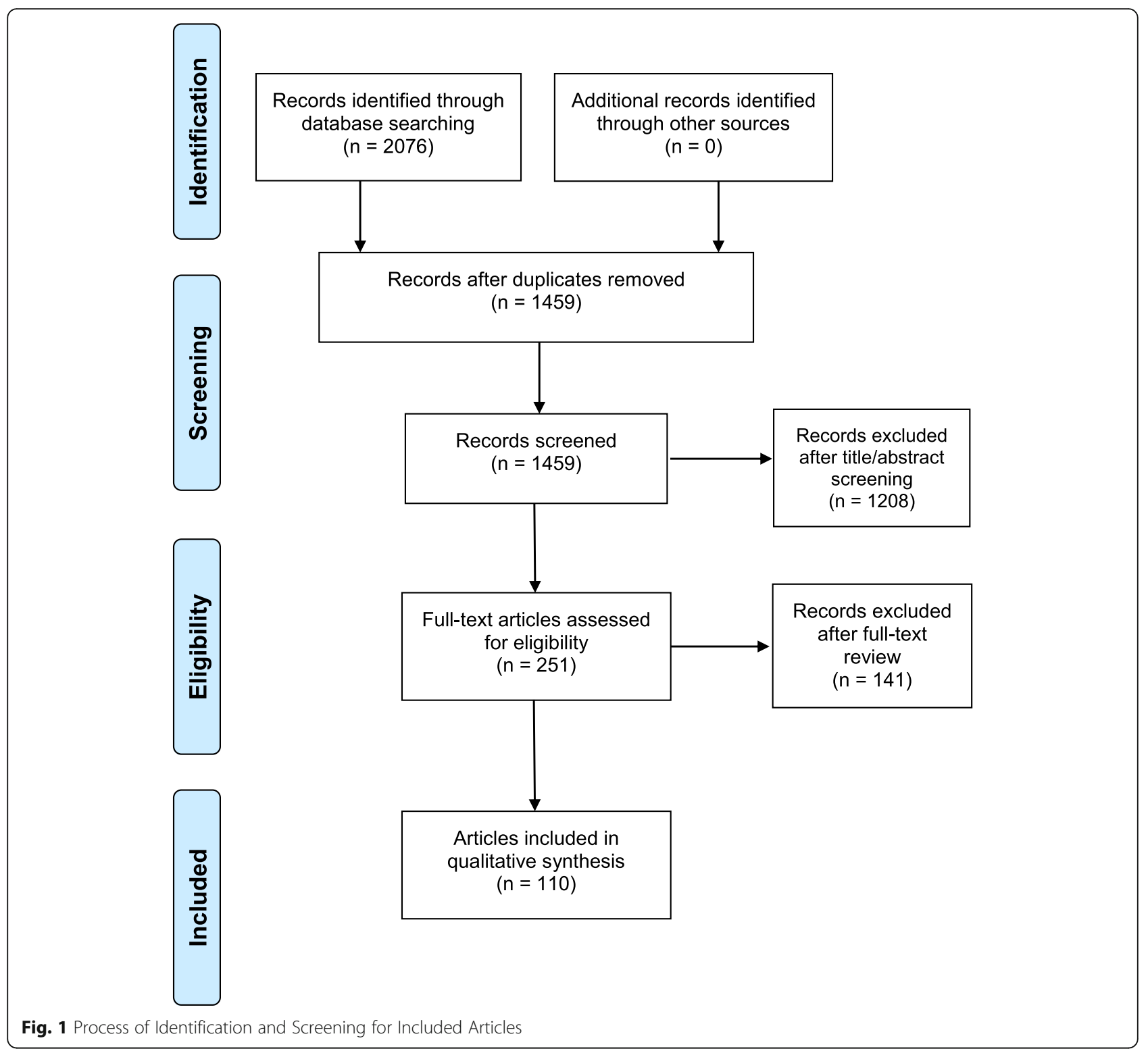

1208 articles were excluded. Full-text review of the remaining 251 articles led to an additional 141 exclusions. A total of 110 articles reporting 144 studies of independent samples were identified as eligible for inclusion in this review. Among those 110 articles, 26 reported two independent samples, one reported three independent samples, and two reported four independent samples to examine physical activity behaviors between sex, age group, or region, or examined both seasonality and weather [28-44].

Table 1 summarizes participant characteristics and study designs for all included articles. In total, articles included 118,189 participants $(62.0 \%$ female participants, median $N=272$, IQR $=85-722$ ) from 30 unique countries on five continents. Figure 2 summarizes the sampling density across the globe. Most data represented western countries such as the United Kingdom, United States, Norway, Australia, Denmark, and Canada. Studies were primarily conducted in countries that the World Bank currently (July 2020) classifies as high income (27/ $30,90.0 \%)$ and three were upper-middle income $(3 / 30$, $10.0 \%$ [45]. None were from low- or middle-income countries.

Approximately equal numbers of studies examined weather $(77 / 144,53.5 \%)$ and seasonality (67/144, 46.5\%). Studies sampled youth (persons under age $18 ; 73 / 144,50.7 \%)$, adults $(44 / 144,30.6 \%)$, and older adults (typically defined as over age 65; 27/144, $18.8 \%)$. Study designs were longitudinal (97/144, $67.4 \%)$ and cross-sectional (47/144, 32.6\%). The modal 
Table 1 Sample and design characteristics of eligible articles

\begin{tabular}{|c|c|c|c|c|c|c|c|c|c|}
\hline Reference & Country & $N$ & $\begin{array}{l}\text { Female } \\
(\%)\end{array}$ & $\begin{array}{l}\text { Age } \\
\text { Group }\end{array}$ & $\begin{array}{l}\text { Age }(M \pm S D \\
\text { [years]) }\end{array}$ & Race & $\begin{array}{l}\text { Monitoring } \\
\text { Period }\end{array}$ & Design & $\begin{array}{l}\text { Environmental } \\
\text { Measure }\end{array}$ \\
\hline $\begin{array}{l}\text { Aadland } \\
\text { et al. (2018) }\end{array}$ & Norway & 465 & 51.6 & Youth & $10.9 \pm 0.3$ & NA & 7 days & Longitudinal & Season \\
\hline $\begin{array}{l}\text { Aebi et al. } \\
(2020)\end{array}$ & Switzerland & 1314 & 48.7 & Adults & $67.9 \pm 7.9$ & NA & 8 days & $\begin{array}{l}\text { Cross- } \\
\text { sectional }\end{array}$ & Season \\
\hline $\begin{array}{l}\text { Aibar } \\
\text { Solana et al. } \\
(2015)\end{array}$ & France, Spain & 646 & 58.8 & Youth & $14.3 \pm 0.7$ & NA & 7 days & Longitudinal & Weather \\
\hline $\begin{array}{l}\text { Akande } \\
\text { et al. (2019) }\end{array}$ & Canada & 272 & 43.8 & Adults & $34.9 \pm 12.6$ & $\begin{array}{l}\text { Inuit: } 74.6 \% \text {; Other: } \\
25.4 \%\end{array}$ & 7 days & Longitudinal & \\
\hline $\begin{array}{l}\text { Albrecht } \\
\text { et al. (2020) }\end{array}$ & Germany & 577 & 52.0 & $\begin{array}{l}\text { Older } \\
\text { adults }\end{array}$ & Range: 65-75 & NA & 7 days & Longitudinal & \\
\hline $\begin{array}{l}\text { Al- } \\
\text { Mohannadi } \\
\text { et al. (2016) }\end{array}$ & Qatar & 2088 & 33.4 & Adults & $41.6 \pm 10.7$ & $\begin{array}{l}\text { Eastern Mediterranean: } \\
\text { 52.3\%; South East } \\
\text { Asian: 30.7\%; Western } \\
\text { Pacific:8.0\%; African: } \\
\text { 3.4\%; North/South } \\
\text { American: } 3.1 \% \text {; } \\
\text { European: } 2.5 \%\end{array}$ & 24 months & Longitudinal & \\
\hline $\begin{array}{l}\text { Arnardottir } \\
\text { et al. (2017) }\end{array}$ & Iceland & 138 & 60.1 & $\begin{array}{l}\text { Older } \\
\text { adults }\end{array}$ & $80.3 \pm 4.9$ & NA & 7 days & $\begin{array}{l}\text { Cross- }^{-} \\
\text {sectional }\end{array}$ & Season \\
\hline $\begin{array}{l}\text { Aspvik et al. } \\
\text { (2018) }\end{array}$ & Norway & 1219 & 51.2 & $\begin{array}{l}\text { Older } \\
\text { adults }\end{array}$ & $72.4 \pm 2.1$ & NA & 7 days & Longitudinal & Weather \\
\hline $\begin{array}{l}\text { Atkin et al. } \\
\text { (2016) }\end{array}$ & $\begin{array}{l}\text { United Kingdom, } \\
\text { Wales, Scotland, } \\
\text { Northern Ireland }\end{array}$ & 704 & 52.6 & Youth & $7.6 \pm 0.3$ & $\begin{array}{l}\text { Caucasian: 92.9\%; } \\
\text { Other: } 7.1 \%\end{array}$ & 7 days & Longitudinal & Weather \\
\hline $\begin{array}{l}\text { Badland } \\
\text { et al. (2011) }\end{array}$ & Australia & 1754 & 59.3 & Adults & $39.9 \pm 11.8$ & NA & 7 days & Longitudinal & $\begin{array}{l}\text { Season, } \\
\text { Weather }\end{array}$ \\
\hline $\begin{array}{l}\text { Balish et al. } \\
\text { (2017) }\end{array}$ & Canada & 190 & 45.3 & Adults & NA & NA & 7 days & Longitudinal & $\begin{array}{l}\text { Season, } \\
\text { Weather }\end{array}$ \\
\hline $\begin{array}{l}\text { Barkley and } \\
\text { Herrmann } \\
(2017)\end{array}$ & United States & 16 & NA & $\begin{array}{l}\text { Older } \\
\text { adults }\end{array}$ & NA & NA & 7 days & Longitudinal & Season \\
\hline $\begin{array}{l}\text { Beighle } \\
\text { et al. (2013) }\end{array}$ & United States & 321 & 52.0 & Youth & $9.1 \pm 1.5$ & NA & 7 days & Longitudinal & Season \\
\hline $\begin{array}{l}\text { Bejarano } \\
\text { et al. (2019) }\end{array}$ & United States & 26 & 42.3 & Youth & $16 \pm 1.6$ & $\begin{array}{l}\text { Caucasian: 69.2\%; } \\
\text { Native American: } \\
\text { 15.4\%; Hispanic: 11.5\%; } \\
\text { Asian: } 3.8 \%\end{array}$ & 20 days & Longitudinal & Weather \\
\hline $\begin{array}{l}\text { Boutou } \\
\text { et al. (2019) }\end{array}$ & $\begin{array}{l}\text { United Kingdom, } \\
\text { Belgium, Greece, } \\
\text { Netherlands }\end{array}$ & 157 & 75.8 & Adults & $67.2 \pm 7.8$ & NA & 7 days & Longitudinal & Weather \\
\hline $\begin{array}{l}\text { Brandon } \\
\text { et al. (2009) }\end{array}$ & Canada & 48 & 75.0 & $\begin{array}{l}\text { Older } \\
\text { adults }\end{array}$ & $77.4 \pm 4.7$ & NA & 7 days & Longitudinal & Weather \\
\hline $\begin{array}{l}\text { Bremer } \\
\text { et al. (2019) }\end{array}$ & Canada & 110 & 51.8 & Youth & $10.2 \pm 1.7$ & NA & 7 days & Longitudinal & Weather \\
\hline $\begin{array}{l}\text { Bringolf-Isler } \\
\text { et al. (2009) }\end{array}$ & Switzerland & 164 & 52.0 & Youth & Range: 6-14 & NA & 7 days & Longitudinal & Season \\
\hline $\begin{array}{l}\text { Brychta } \\
\text { et al. (2016) }\end{array}$ & Iceland & 70 & 64.3 & $\begin{array}{l}\text { Older } \\
\text { adults }\end{array}$ & $79.5 \pm 4.8$ & NA & 7 days & $\begin{array}{l}\text { Cross- } \\
\text { sectional }\end{array}$ & Weather \\
\hline $\begin{array}{l}\text { Buchowski } \\
\text { et al. (2009) }\end{array}$ & United States & 57 & 100 & Adults & $36.5 \pm 9.2$ & NA & 7 days & Longitudinal & Season \\
\hline $\begin{array}{l}\text { Button et al. } \\
(2020)\end{array}$ & Canada & 90 & 61.1 & Youth & $10.6 \pm 1.4$ & $\begin{array}{l}\text { White: } 56.7 \% \text {; } \\
\text { Indigenous \& Visible } \\
\text { Minority: } 43.3 \%\end{array}$ & 8 days & Longitudinal & Weather \\
\hline $\begin{array}{l}\text { Carr et al. } \\
\text { (2016) }\end{array}$ & United States & 132 & 100 & Adults & $41.6 \pm 10.1$ & Hispanic/Latino: 100\% & 12 months & Longitudinal & Season \\
\hline
\end{tabular}


Table 1 Sample and design characteristics of eligible articles (Continued)

\begin{tabular}{|c|c|c|c|c|c|c|c|c|c|}
\hline Reference & Country & $N$ & $\begin{array}{l}\text { Female } \\
(\%)\end{array}$ & $\begin{array}{l}\text { Age } \\
\text { Group }\end{array}$ & $\begin{array}{l}\text { Age }(M \pm S D \\
\text { [years] })\end{array}$ & Race & $\begin{array}{l}\text { Monitoring } \\
\text { Period }\end{array}$ & Design & $\begin{array}{l}\text { Environmental } \\
\text { Measure }\end{array}$ \\
\hline $\begin{array}{l}\text { Cepeda } \\
\text { et al. (2018) }\end{array}$ & Netherlands & 1166 & 44.4 & $\begin{array}{l}\text { Adults } \\
\& \\
\text { older } \\
\text { adults }\end{array}$ & $\begin{array}{l}\text { Middle aged: } \\
\text { Median = 59.1 } \\
\text { [IQR = 56.1-62.4]; } \\
\text { Young elderly: } \\
71.3 \text { [67.3-72.8]; } \\
\text { Old elderly: } 78.9 \\
\text { [77.0-81.4] }\end{array}$ & NA & 7 days & $\begin{array}{l}\text { Cross- } \\
\text { sectional }\end{array}$ & Season \\
\hline $\begin{array}{l}\text { Chang et al. } \\
(2020)\end{array}$ & China & 53 & 49.1 & Youth & $4.9 \pm 0.2$ & NA & 7 days & Longitudinal & Season \\
\hline $\begin{array}{l}\text { Clemes } \\
\text { et al. (2011) }\end{array}$ & United Kingdom & 96 & 53.2 & Adults & $40.7 \pm 12.5$ & NA & 4 weeks & Longitudinal & Season \\
\hline $\begin{array}{l}\text { Collings } \\
\text { et al. (2020) }\end{array}$ & United Kingdom & 342 & 48.8 & Youth & $3.4 \pm 0.8$ & $\begin{array}{l}\text { White: } 40.9 \% \text {; South } \\
\text { Asian: } 59.1 \%\end{array}$ & 6 days & Longitudinal & Season \\
\hline $\begin{array}{l}\text { Colom et al. } \\
\text { (2019) }\end{array}$ & Spain & 218 & 51.0 & Adults & Range: 55-75 & NA & 9 days & $\begin{array}{l}\text { Cross- } \\
\text { sectional }\end{array}$ & Weather \\
\hline $\begin{array}{l}\text { Cooper } \\
\text { et al. (2010) }\end{array}$ & United Kingdom & 1010 & 53.3 & Youth & $11.0 \pm 0.4$ & NA & 4 days & $\begin{array}{l}\text { Cross- } \\
\text { sectional }\end{array}$ & Season \\
\hline $\begin{array}{l}\text { Cradock } \\
\text { et al. (2009) }\end{array}$ & United States & 152 & 41.0 & Youth & $13.7 \pm 0.7$ & $\begin{array}{l}\text { White: } 57.0 \% \text {; Black } \\
\text { African-American: } \\
\text { 10.0\%; Hispanic: 13.0\%; } \\
\text { Asian: 12.0\%; Other } \\
\text { Race/Ethnicity: } 9.0 \%\end{array}$ & 4 days & $\begin{array}{l}\text { Cross- } \\
\text { sectional }\end{array}$ & Weather \\
\hline $\begin{array}{l}\text { Crowley } \\
\text { et al. (2016) }\end{array}$ & United States & 510 & 55.0 & Adults & $43.5 \pm 9.2$ & NA & 12 months & Longitudinal & Season \\
\hline $\begin{array}{l}\text { Cullen et al. } \\
\text { (2017) }\end{array}$ & United States & 342 & 100 & Youth & Range: 8-10 & $\begin{array}{l}\text { African-American: } \\
100 \%\end{array}$ & 7 days & $\begin{array}{l}\text { Cross- }^{-} \\
\text {sectional }\end{array}$ & Weather \\
\hline $\begin{array}{l}\text { Davis et al. } \\
\text { (2011) }\end{array}$ & United Kingdom & 230 & 49.1 & $\begin{array}{l}\text { Older } \\
\text { adults }\end{array}$ & $78.1 \pm 5.8$ & & 7 days & Longitudinal & $\begin{array}{l}\text { Season, } \\
\text { Weather }\end{array}$ \\
\hline $\begin{array}{l}\text { de Vries } \\
\text { et al. (2019) }\end{array}$ & Netherlands & 10 & 50.0 & Youth & 12.5 & NA & 14 days & Longitudinal & $\begin{array}{l}\text { Season, } \\
\text { Weather }\end{array}$ \\
\hline $\begin{array}{l}\text { Declòs-Alió } \\
\text { et al. (2019) }\end{array}$ & Spain & 227 & 56.0 & $\begin{array}{l}\text { Older } \\
\text { adults }\end{array}$ & NA & NA & 7 days & Longitudinal & Season \\
\hline $\begin{array}{l}\text { Deng and } \\
\text { Fredriksen } \\
(2018)\end{array}$ & Norway & 2123 & 49.6 & Youth & $9 \pm 1.5$ & NA & 7 days & $\begin{array}{l}\text { Cross- } \\
\text { sectional }\end{array}$ & Season \\
\hline $\begin{array}{l}\text { Dias et al. } \\
\text { (2019) }\end{array}$ & $\begin{array}{l}\text { Switzerland, } \\
\text { Belgium, United } \\
\text { Kingdom, United } \\
\text { States }\end{array}$ & 1052 & 49.8 & Youth & $3.7 \pm 0.4$ & NA & 3 days & Longitudinal & $\begin{array}{l}\text { Season, } \\
\text { Weather }\end{array}$ \\
\hline $\begin{array}{l}\text { Diaz et al. } \\
\text { (2016) }\end{array}$ & United States & 2096 & 54.2 & Adults & Range: $45-75$ & Black: $31.6 \%$ & 7 days & $\begin{array}{l}\text { Cross- } \\
\text { sectional }\end{array}$ & Season \\
\hline $\begin{array}{l}\text { Dill et al. } \\
\text { (2014) }\end{array}$ & United States & 353 & NA & Adults & NA & & 3 days & Longitudinal & Weather \\
\hline $\begin{array}{l}\text { Duncan } \\
\text { et al. (2008) }\end{array}$ & New Zealand & 1115 & 51.9 & Youth & Range: 5-16 & $\begin{array}{l}\text { Caucasian: } 49.2 \% ; \\
\text { Polynesian: 30.0\%; } \\
\text { Asian: 16.5\%; Other: } \\
4.3 \%\end{array}$ & 5 days & $\begin{array}{l}\text { Cross- } \\
\text { sectional }\end{array}$ & Season \\
\hline $\begin{array}{l}\text { Edwards } \\
\text { et al. (2015) }\end{array}$ & United States & 372 & 48.0 & Youth & $3.4 \pm 0.3$ & $\begin{array}{l}\text { Caucasian: } 88.0 \% \\
\text { African-American: } \\
22.0 \%\end{array}$ & 3 days & Longitudinal & Season \\
\hline $\begin{array}{l}\text { Feinglass } \\
\text { et al. (2011) }\end{array}$ & United States & 241 & 74.7 & Adults & $\begin{array}{l}<50 \text { years: } \\
(26.1 \%) ; 50-65 \\
\text { years: }(39.3 \%) ; 66- \\
75 \text { years: }(22.2 \%) ; \\
>75 \text { years: }(12.5 \%)\end{array}$ & $\begin{array}{l}\text { Caucasian/ Other: } \\
\text { 75.1\%; African- } \\
\text { American: 17.8\%; } \\
\text { Hispanic/ Latino: 7.1\% }\end{array}$ & 7 days & Longitudinal & Weather \\
\hline $\begin{array}{l}\text { Goodman } \\
\text { et al. (2012) }\end{array}$ & United Kingdom & 325 & 52.3 & Youth & Range: 8-11 & NA & 4 days & Longitudinal & Weather \\
\hline
\end{tabular}


Table 1 Sample and design characteristics of eligible articles (Continued)

\begin{tabular}{|c|c|c|c|c|c|c|c|c|c|}
\hline Reference & Country & $N$ & $\begin{array}{l}\text { Female } \\
(\%)\end{array}$ & $\begin{array}{l}\text { Age } \\
\text { Group }\end{array}$ & $\begin{array}{l}\text { Age }(M \pm S D \\
\text { [years]) }\end{array}$ & Race & $\begin{array}{l}\text { Monitoring } \\
\text { Period }\end{array}$ & Design & $\begin{array}{l}\text { Environmental } \\
\text { Measure }\end{array}$ \\
\hline $\begin{array}{l}\text { Goodman } \\
\text { et al. (2014) }\end{array}$ & $\begin{array}{l}\text { Australia, Brazil, } \\
\text { Denmark, } \\
\text { England, Estonia, } \\
\text { Spain, Norway, } \\
\text { Switzerland, } \\
\text { United States }\end{array}$ & $\begin{array}{l}23 \\
188\end{array}$ & 62.0 & Youth & Range: 5-16 & NA & 7 days & Longitudinal & Weather \\
\hline $\begin{array}{l}\text { Gracia- } \\
\text { Marco et al. } \\
\text { (2013) }\end{array}$ & $\begin{array}{l}\text { Sweden, Greece, } \\
\text { Italy, Spain, } \\
\text { Hungary, } \\
\text { Belgium, France, } \\
\text { Germany, Austria }\end{array}$ & 2173 & 54.0 & Youth & $15 \pm 1.2$ & NA & 7 days & Longitudinal & Season \\
\hline $\begin{array}{l}\text { Griew et al. } \\
\text { (2010) }\end{array}$ & United Kingdom & 1307 & 51.8 & Youth & Range: 10-11 & NA & 5 days & Longitudinal & Weather \\
\hline $\begin{array}{l}\text { Hagströmer } \\
\text { et al. (2014) }\end{array}$ & Sweden & 1172 & 54.0 & Adults & $45 \pm 15$ & NA & 7 days & Longitudinal & Season \\
\hline $\begin{array}{l}\text { Hamilton } \\
\text { et al. (2009) }\end{array}$ & United Kingdom & 96 & 51.1 & Adults & $41.0 \pm 12.3$ & NA & 4 weeks & Longitudinal & Weather \\
\hline $\begin{array}{l}\text { Harrison } \\
\text { et al. (2011) }\end{array}$ & United Kingdom & 1794 & 55.0 & Youth & Range: 9-10 & NA & 7 days & Longitudinal & Weather \\
\hline $\begin{array}{l}\text { Harrison } \\
\text { et al. (2015) }\end{array}$ & United Kingdom & 283 & 55.1 & Youth & Range: 9-14 & NA & 7 days & Longitudinal & Weather \\
\hline $\begin{array}{l}\text { Harrison } \\
\text { et al. (2017) }\end{array}$ & $\begin{array}{l}\text { United Kingdom, } \\
\text { Switzerland, } \\
\text { Belgium, } \\
\text { Australia, } \\
\text { Denmark, } \\
\text { Estonia, Norway, } \\
\text { Madeira, United } \\
\text { States }\end{array}$ & $\begin{array}{l}23 \\
451\end{array}$ & 62.0 & Youth & Range: 3-18 & NA & 7 days & Longitudinal & Weather \\
\hline $\begin{array}{l}\text { Hjorth et al. } \\
\text { (2013) }\end{array}$ & Denmark & 730 & 48.5 & Youth & $10.0 \pm 0.6$ & NA & 7 days & Longitudinal & Weather \\
\hline $\begin{array}{l}\text { Hoaas et al. } \\
\text { (2019) }\end{array}$ & $\begin{array}{l}\text { Norway, } \\
\text { Denmark, } \\
\text { Australia }\end{array}$ & 168 & 42.9 & Adults & Range: 60-73 & NA & 7 days & Longitudinal & Season \\
\hline $\begin{array}{l}\text { Hopkins } \\
\text { et al. (2011) }\end{array}$ & United States & 145 & 59.3 & Youth & $10.7 \pm 0.3$ & NA & 7 days & Longitudinal & Season \\
\hline $\begin{array}{l}\text { Hoppmann } \\
\text { et al. (2017) }\end{array}$ & Canada & 126 & 64.0 & $\begin{array}{l}\text { Older } \\
\text { adults }\end{array}$ & $71.9 \pm 5$ & $\begin{array}{l}\text { European: } 62.0 \% \text {; Asian: } \\
\text { 36.0\%; Other: 1.0\%; } \\
\text { Missing: } 1.0 \%\end{array}$ & 10 days & Longitudinal & Weather \\
\hline $\begin{array}{l}\text { Hunter et al. } \\
\text { (2019) }\end{array}$ & Canada & 149 & 47.0 & Adults & $19 \pm 1.9$ & $\begin{array}{l}\text { White: } 59.7 \% \text {; Other: } \\
40.3 \%\end{array}$ & 7 days & Longitudinal & Weather \\
\hline $\begin{array}{l}\text { Jehn et al. } \\
\text { (2014) }\end{array}$ & Germany & 15 & 40.0 & Adults & $66.7 \pm 5.2$ & NA & 6 months & Longitudinal & Season \\
\hline $\begin{array}{l}\text { Jones et al. } \\
\text { (2017) }\end{array}$ & Canada & 42 & 24.0 & $\begin{array}{l}\text { Older } \\
\text { adults }\end{array}$ & $77.4 \pm 4.7$ & NA & 7 days & $\begin{array}{l}\text { Cross- } \\
\text { sectional }\end{array}$ & Weather \\
\hline $\begin{array}{l}\text { Jones et al. } \\
\text { (2017) }\end{array}$ & Canada & 42 & NA & $\begin{array}{l}\text { Older } \\
\text { adults }\end{array}$ & $77.4 \pm 4.7$ & NA & 7 days & Longitudinal & $\begin{array}{l}\text { Season, } \\
\text { Weather }\end{array}$ \\
\hline $\begin{array}{l}\text { Katapally } \\
\text { et al. (2016) }\end{array}$ & Canada & 331 & 49.8 & Youth & $11.6 \pm 1.1$ & NA & 7 days & Longitudinal & Weather \\
\hline $\begin{array}{l}\text { Kharlova } \\
\text { et al. (2020) }\end{array}$ & Norway & 2015 & 50.6 & Youth & $9.5 \pm 1.8$ & NA & 6 days & Longitudinal & Season \\
\hline $\begin{array}{l}\text { Kimura et al. } \\
\text { (2015) }\end{array}$ & Japan & 39 & 56.0 & $\begin{array}{l}\text { Older } \\
\text { adults }\end{array}$ & $70.7 \pm 3.2$ & NA & 2 weeks & Longitudinal & Weather \\
\hline $\begin{array}{l}\text { King et al. } \\
\text { (2011) }\end{array}$ & United Kingdom & 480 & 49.1 & Youth & NA & NA & 7 days & Longitudinal & Season \\
\hline Kolle et al. & Norway & 1824 & 48.5 & Youth & Range: 9-15 & NA & 4 days & Cross- & Weather \\
\hline
\end{tabular}


Table 1 Sample and design characteristics of eligible articles (Continued)

\begin{tabular}{|c|c|c|c|c|c|c|c|c|c|}
\hline Reference & Country & $N$ & $\begin{array}{l}\text { Female } \\
(\%)\end{array}$ & $\begin{array}{l}\text { Age } \\
\text { Group }\end{array}$ & $\begin{array}{l}\text { Age }(M \pm S D \\
\text { [years]) }\end{array}$ & Race & $\begin{array}{l}\text { Monitoring } \\
\text { Period }\end{array}$ & Design & $\begin{array}{l}\text { Environmental } \\
\text { Measure }\end{array}$ \\
\hline$(2009)$ & & & & & & & & sectional & \\
\hline $\begin{array}{l}\text { Kong et al. } \\
(2020)\end{array}$ & South Korea & 555 & 43.8 & Adults & $61.1 \pm 8.9$ & NA & 7 days & $\begin{array}{l}\text { cross- } \\
\text { sectional }\end{array}$ & Weather \\
\hline $\begin{array}{l}\text { Koolhaas } \\
\text { et al. (2017) }\end{array}$ & Netherlands & 1200 & 52.3 & $\begin{array}{l}\text { Older } \\
\text { adults }\end{array}$ & $77.5 \pm 5.0$ & NA & 7 days & $\begin{array}{l}\text { Cross- } \\
\text { sectional }\end{array}$ & Season \\
\hline $\begin{array}{l}\text { Larouche } \\
\text { et al. (2019) }\end{array}$ & Canada & 1699 & 55.0 & Youth & $10.2 \pm 1.0$ & NA & 8 days & $\begin{array}{l}\text { Cross- } \\
\text { sectional }\end{array}$ & Season \\
\hline $\begin{array}{l}\text { Lewis et al. } \\
\text { (2016) }\end{array}$ & $\begin{array}{l}\text { Australia and } \\
\text { Canada }\end{array}$ & 953 & 57.0 & Youth & $10.6 \pm 0.4$ & NA & 7 days & $\begin{array}{l}\text { Cross- } \\
\text { sectional }\end{array}$ & Season \\
\hline $\begin{array}{l}\text { Ma et al. } \\
(2018)\end{array}$ & Hong Kong & 210 & 33.8 & Adults & $26.1 \pm 8.7$ & NA & 35 days & Longitudinal & Season \\
\hline $\begin{array}{l}\text { Martins } \\
\text { et al. (2015) }\end{array}$ & Brazil & 16 & 50.0 & Adults & Range: 18-35 & NA & 6 days & Longitudinal & Weather \\
\hline $\begin{array}{l}\text { McCrorie } \\
\text { et al. (2015) }\end{array}$ & Scotland & 33 & 54.5 & Youth & $12.2 \pm 0.3$ & NA & 8 days & $\begin{array}{l}\text { Cross- } \\
\text { sectional }\end{array}$ & Weather \\
\hline $\begin{array}{l}\text { Mckee et al. } \\
\text { (2012) }\end{array}$ & Northern Ireland & 85 & 38.8 & Youth & Range: 4-5 & NA & 6 days & Longitudinal & Weather \\
\hline $\begin{array}{l}\text { McMurdo } \\
\text { et al. (2012) }\end{array}$ & United Kingdom & 547 & 54.0 & $\begin{array}{l}\text { Older } \\
\text { adults }\end{array}$ & $79 \pm 8$ & NA & 7 days & $\begin{array}{l}\text { Cross- } \\
\text { sectional }\end{array}$ & Weather \\
\hline $\begin{array}{l}\text { Mitchell } \\
\text { et al. (2018) }\end{array}$ & United States & 575 & 34.3 & Adults & $38.6 \pm 0.1$ & Hispanic: 100.0\% & 1 day & $\begin{array}{l}\text { Cross- } \\
\text { sectional }\end{array}$ & Weather \\
\hline $\begin{array}{l}\text { Mitsui et al. } \\
(2010)\end{array}$ & Japan & 50 & 0.0 & Adults & $43.6 \pm 10.8$ & NA & 7 days & Longitudinal & Weather \\
\hline $\begin{array}{l}\text { Nagy et al. } \\
\text { (2019) }\end{array}$ & United Kingdom & 108 & 51.0 & Youth & $7.5 \pm 0.5$ & $\begin{array}{l}\text { Caucasian: 59.0\%; } \\
\text { South Asian: } 41.0 \%\end{array}$ & 7 days & $\begin{array}{l}\text { Cross- } \\
\text { sectional }\end{array}$ & Season \\
\hline $\begin{array}{l}\text { Nakashima } \\
\text { et al. (2019) }\end{array}$ & Japan & 22 & 86.4 & $\begin{array}{l}\text { Older } \\
\text { adults }\end{array}$ & $75.1 \pm 7.3$ & NA & 7 days & Longitudinal & Season \\
\hline $\begin{array}{l}\text { Newman } \\
\text { et al. (2009) }\end{array}$ & United States & 500 & 100.0 & Adults & $57 \pm 2.9$ & $\begin{array}{l}\text { African-American: } \\
13.2 \%\end{array}$ & 7 days & $\begin{array}{l}\text { Cross- } \\
\text { sectional }\end{array}$ & Season \\
\hline $\begin{array}{l}\text { Nilsen et al. } \\
\text { (2019) }\end{array}$ & Norway & 1154 & 49.0 & Youth & $4.7 \pm 0.9$ & NA & 14 days & Longitudinal & Season \\
\hline $\begin{array}{l}\text { O'Connell } \\
\text { et al. (2013) }\end{array}$ & United Kingdom & 46 & 72.0 & Adults & $41.7 \pm 14.4$ & NA & 7 days & Longitudinal & Season \\
\hline $\begin{array}{l}\text { Ogawa } \\
\text { et al. (2018) }\end{array}$ & Japan & 35 & 65.7 & Adults & $69.3 \pm 5.3$ & NA & 1 month & Longitudinal & Season \\
\hline $\begin{array}{l}\text { Oliver et al. } \\
\text { (2011) }\end{array}$ & New Zealand & 135 & 60.0 & Youth & Range: 5-6 & NA & 8 days & $\begin{array}{l}\text { Cross- } \\
\text { sectional }\end{array}$ & Season \\
\hline $\begin{array}{l}\text { Pagels et al. } \\
\text { (2016) }\end{array}$ & Sweden & 179 & 48.6 & Youth & $11.1 \pm 2.1$ & NA & 5 days & Longitudinal & Season \\
\hline $\begin{array}{l}\text { Patnode } \\
\text { et al. (2010) }\end{array}$ & United States & 294 & 49.3 & Youth & $15.4 \pm 1.7$ & $\begin{array}{l}\text { Caucasian: 93.5\%; } \\
\text { Other: } 6.5 \%\end{array}$ & 7 days & $\begin{array}{l}\text { Cross- } \\
\text { sectional }\end{array}$ & Season, weather \\
\hline $\begin{array}{l}\text { Pearce et al. } \\
\text { (2012) }\end{array}$ & United Kingdom & 482 & 52.0 & Youth & Range: 8-10 & NA & 7 days & Longitudinal & Season \\
\hline $\begin{array}{l}\text { Pechova } \\
\text { et al. (2019) }\end{array}$ & $\begin{array}{l}\text { Czech Republic, } \\
\text { Slovakia \& } \\
\text { Poland }\end{array}$ & 83 & 89.7 & Adults & $65 \pm 6$ & NA & 8 days & Longitudinal & $\begin{array}{l}\text { Season, } \\
\text { Weather }\end{array}$ \\
\hline $\begin{array}{l}\text { Pelclova } \\
\text { et al. (2010) }\end{array}$ & Czech Republic & 13 & 84.6 & Youth & $15.6 \pm 0.5$ & NA & 10 months & Longitudinal & Weather \\
\hline $\begin{array}{l}\text { Prins and } \\
\text { van Lenthe } \\
\text { (2015) }\end{array}$ & Netherlands & 43 & 52.5 & Adults & NA & NA & 7 days & Longitudinal & Weather \\
\hline $\begin{array}{l}\text { Rahman } \\
\text { et al. (2019) }\end{array}$ & Canada & 972 & 58.0 & Youth & $10.9 \pm 0.4$ & NA & 7 days & $\begin{array}{l}\text { Cross- } \\
\text { sectional }\end{array}$ & Season, weather \\
\hline
\end{tabular}


Table 1 Sample and design characteristics of eligible articles (Continued)

\begin{tabular}{|c|c|c|c|c|c|c|c|c|c|}
\hline Reference & Country & $\mathbf{N}$ & $\begin{array}{l}\text { Female } \\
(\%)\end{array}$ & $\begin{array}{l}\text { Age } \\
\text { Group }\end{array}$ & $\begin{array}{l}\text { Age }(M \pm S D \\
\text { [years]) }\end{array}$ & Race & $\begin{array}{l}\text { Monitoring } \\
\text { Period }\end{array}$ & Design & $\begin{array}{l}\text { Environmental } \\
\text { Measure }\end{array}$ \\
\hline $\begin{array}{l}\text { Remmers } \\
\text { et al. (2017) }\end{array}$ & Australia & 307 & 52.0 & Youth & $11.1 \pm 0.7$ & NA & 7 days & Longitudinal & $\begin{array}{l}\text { Season, } \\
\text { Weather }\end{array}$ \\
\hline $\begin{array}{l}\text { Ridgers } \\
\text { et al. (2015) }\end{array}$ & Australia & 326 & 50.3 & Youth & $10 \pm 0.7$ & NA & 7 days & $\begin{array}{l}\text { Cross- } \\
\text { sectional }\end{array}$ & Season \\
\hline $\begin{array}{l}\text { Ridgers } \\
\text { et al. (2018) }\end{array}$ & Australia & 326 & 50.3 & Youth & Range: 8-11 & NA & 7 days & Longitudinal & Season \\
\hline $\begin{array}{l}\text { Robbins } \\
\text { et al. (2013) }\end{array}$ & Canada & 38 & 26.3 & Adults & $54 \pm 7.0$ & NA & 7 days & $\begin{array}{l}\text { Cross- } \\
\text { sectional }\end{array}$ & Weather \\
\hline $\begin{array}{l}\text { Rosenthal } \\
\text { et al. (2020) }\end{array}$ & United States & 266 & 58.4 & Adults & $52.1 \pm 14$ & NA & NA & $\begin{array}{l}\text { Cross- } \\
\text { sectional }\end{array}$ & Weather \\
\hline $\begin{array}{l}\text { Rowlands } \\
\text { et al. (2009) }\end{array}$ & United Kingdom & 64 & 50.0 & Youth & $9.9 \pm 0.3$ & NA & 6 days & Longitudinal & Season \\
\hline $\begin{array}{l}\text { Sartini et al. } \\
\text { (2017) }\end{array}$ & United Kingdom & 1361 & 0.0 & $\begin{array}{l}\text { Older } \\
\text { adults }\end{array}$ & $78.5 \pm 4.6$ & NA & 7 days & Longitudinal & Weather \\
\hline $\begin{array}{l}\text { Schepps } \\
\text { et al. (2018) }\end{array}$ & United States & $\begin{array}{l}16 \\
741\end{array}$ & 100.0 & $\begin{array}{l}\text { Older } \\
\text { adults }\end{array}$ & $72.0 \pm 5.7$ & NA & 7 days & $\begin{array}{l}\text { Cross- } \\
\text { sectional }\end{array}$ & Weather \\
\hline $\begin{array}{l}\text { Sewell et al. } \\
(2010)\end{array}$ & United Kingdom & 95 & 41.0 & Adults & $65.5 \pm 8.5$ & NA & 2 days & $\begin{array}{l}\text { Cross- } \\
\text { sectional }\end{array}$ & Season \\
\hline $\begin{array}{l}\text { Shen et al. } \\
\text { (2013) }\end{array}$ & United States & 46 & 56.5 & Youth & $4.2 \pm 0.2$ & $\begin{array}{l}\text { Caucasian: 68.0\%; } \\
\text { Other: } 32.0 \%\end{array}$ & 5 days & $\begin{array}{l}\text { Cross- } \\
\text { sectional }\end{array}$ & Weather \\
\hline $\begin{array}{l}\text { Silva et al. } \\
\text { (2011) }\end{array}$ & Portugal & 24 & 50.0 & Youth & $11.0 \pm 1.5$ & NA & 7 days & Longitudinal & Season \\
\hline $\begin{array}{l}\text { Sit et al. } \\
(2019)\end{array}$ & Hong Kong & 270 & 40.0 & Youth & NA & NA & 3 days & Longitudinal & Season \\
\hline $\begin{array}{l}\text { Stabell et al. } \\
(2020)\end{array}$ & United States & 38 & 10.5 & Adults & Range: 50-75 & $\begin{array}{l}\text { Caucasian:81.6\%; Other: } \\
18.4 \%\end{array}$ & 24 weeks & Longitudinal & Weather \\
\hline $\begin{array}{l}\text { Sugino } \\
\text { et al. (2012) }\end{array}$ & Japan & 9 & 0.0 & Adults & $71.7 \pm 8.3$ & NA & 2 weeks & $\begin{array}{l}\text { Cross- } \\
\text { sectional }\end{array}$ & Season \\
\hline $\begin{array}{l}\text { Sumukadas } \\
\text { et al. (2008) }\end{array}$ & Scotland & 127 & 71.0 & $\begin{array}{l}\text { Older } \\
\text { adults }\end{array}$ & $78.6 \pm 6.5$ & NA & 7 days & Longitudinal & Season \\
\hline $\begin{array}{l}\text { Van Kann } \\
\text { et al. (2016) }\end{array}$ & Netherlands & 520 & 55.6 & Youth & $10.1 \pm 0.7$ & NA & 7 days & Longitudinal & Weather \\
\hline $\begin{array}{l}\text { Wang et al. } \\
\text { (2017) }\end{array}$ & China & 34 & 38.2 & Adults & $31 \pm 10$ & NA & 7 days & Longitudinal & Weather \\
\hline $\begin{array}{l}\text { Witham } \\
\text { et al. (2014) }\end{array}$ & United Kingdom & 547 & 54.3 & $\begin{array}{l}\text { Older } \\
\text { adults }\end{array}$ & $78.5 \pm 7.7$ & NA & 7 days & Longitudinal & Weather \\
\hline $\begin{array}{l}\text { Wong et al. } \\
(2020)\end{array}$ & Mexico & 559 & 49.0 & Youth & $4.8 \pm 0.5$ & NA & 7 days & Longitudinal & Weather \\
\hline $\begin{array}{l}\text { Wu et al. } \\
\text { (2017) }\end{array}$ & United Kingdom & 4051 & 55.7 & Adults & $67.4 \pm 9.5$ & NA & 7 days & $\begin{array}{l}\text { Cross- } \\
\text { sectional }\end{array}$ & Weather \\
\hline $\begin{array}{l}\text { Yildrim et al. } \\
\text { (2012) }\end{array}$ & $\begin{array}{l}\text { Belgium, Greece, } \\
\text { Hungary, } \\
\text { Netherlands, } \\
\text { Switzerland }\end{array}$ & 722 & 53.0 & Youth & $11.6 \pm 0.9$ & NA & 6 days & $\begin{array}{l}\text { Cross- } \\
\text { sectional }\end{array}$ & Weather \\
\hline $\begin{array}{l}\text { Zheng et al. } \\
\text { (2019) }\end{array}$ & Hong Kong & 740 & 40.9 & Youth & $14.7 \pm 1.6$ & Chinese: $100.0 \%$ & 7 days & Longitudinal & Weather \\
\hline
\end{tabular}




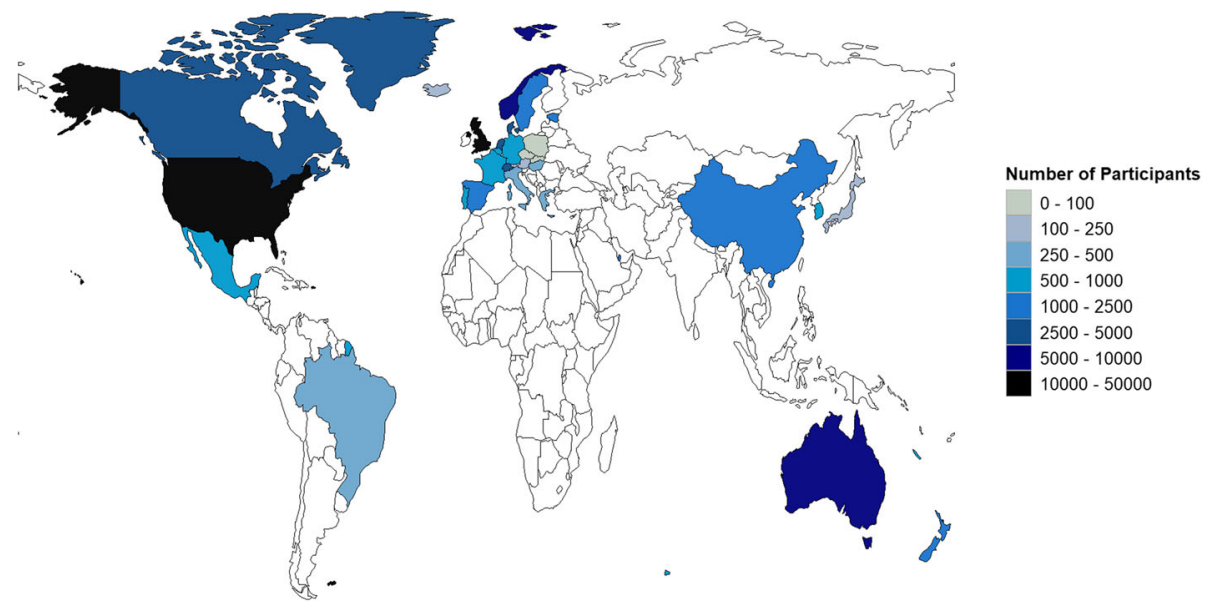

Fig. 2 Geographic distribution of participants included in this review

duration of activity monitoring for movement behavior was 7 days $(82 / 144,56.9 \%)$ but ranged from 1 day to 2 years.

\section{Activity monitoring methods}

Almost all studies measured physical activity (138/144, 95.2\%) and fewer measured sedentary behavior (50/144, $34.7 \%)$. Table 2 summarizes characteristics of the devices used to measure physical activity and sedentary behavior. Physical activity was measured with research-grade accelerometers $(121 / 144,84.0 \%)$, pedometers $(22 / 144$, $15.3 \%)$, or global positioning system logger (1/144, $0.7 \%)$. Although most studies with accelerometer-based measurements used wearable devices, one study measured physical activity using the accelerometer contained within a smartphone [46].

Physical activity measures included volume (i.e., step counts, total accelerometer counts) and intensity-specific durations. Volume measures represent the total amount of energy expended in physical activity whereas intensity-specific durations represent how individuals allocate their time to more and less effortful forms of physical activity. Figure 3 summarizes the frequency of studies using volume and intensity-specific duration measures in samples of youth, adults, and older adults. Seasonal differences in physical activity were more frequently estimated in youth than adults or older adults. Weather indices associated with physical activity were studied most frequently in youth, and approximately equally in adults and older adults.

Sedentary behavior was typically classified using accelerometer cut-points that included $<50,<100, \leq 100$, < $150,<175$, and $<200$ counts per minute $[33,38,39,47-$ 70]. Other sedentary behavior classifications were based on accumulating less than 122 counts per minute, less than or equal to 328 counts per minute [71], less than
800 vector magnitude counts per minute [72], less than 820 vector magnitude counts per minute [73, 74], less than 1110 vector magnitude counts per minute $[75,76]$, falling below activity registering at 1.0 [77] or 1.5 [7880] metabolic equivalents to task (METs). Four studies in three papers did not report how sedentary behavior was defined [30, 34, 81].

\section{Seasonal differences in physical activity and movement- related behaviors}

Table 3 summarizes seasonal differences in a variety of movement-related behaviors. Studies either compared two seasons $(29 / 67,43.3 \%)$, three seasons $(9 / 67,13.4 \%)$, or four seasons $(25 / 67,37.3 \%)$. Four studies did not report their definition of season $(4 / 67,6.0 \%)[86,87,103]$. Winter $(64 / 67,95.5 \%)$ and summer $(51 / 67,76.1 \%)$ were studied most frequently, with spring $(40 / 67,59.7 \%)$ and autumn $(36 / 67,53.7 \%)$ receiving less attention. Studies comparing physical activity and sedentary behavior between seasons sampled youth $(40 / 67,59.7 \%)$, adults (17/ $67,25.4 \%)$ and older adults (10/67, 14.9\%). Studies of seasonal differences measured volume (47/67, 70.1\%), moderate-to-vigorous intensity physical activity (MVPA) duration (37/67, 55.2\%), light-intensity physical activity (LPA) duration $(19 / 67,28.4 \%)$, and sedentary behavior (25/67, 37.3\%).

\section{Physical activity volume}

Studies comparing volume between seasons sampled youth $(29 / 47,61.7 \%)$, adults $(13 / 47,27.7 \%)$ and older adults $(5 / 47,10.6 \%)$. The most common seasonal comparisons of volume involved winter vs. summer (30/47, $63.8 \%)$, winter vs. spring $(22 / 47,46.8 \%)$, spring vs. autumn $(12 / 47,25.5 \%)$, and winter vs. autumn $(12 / 47$, $25.5 \%)$. 
Table 2 Characteristics of devices used to measure physical activity and sedentary behavior

\begin{tabular}{|c|c|c|c|c|}
\hline Reference & $\begin{array}{l}\text { Type of } \\
\text { Device }\end{array}$ & Specific Device & Wear Location & $\begin{array}{l}\text { Measure(s) of } \\
\text { Activity }\end{array}$ \\
\hline Aadland et al. (2018) & Accelerometer & ActiGraph GT3X+ & NA & SB, LPA, MVPA, V \\
\hline Aebi et al. (2020) & Accelerometer & ActiGraph wGT3X-BT & Hip & SB, LPA, MVPA \\
\hline Aibar Solana et al. (2015) & Accelerometer & ActiGraph GT3X & NA & SB \\
\hline Alande et al. (2019) & Pedometer & Kaden G-Sport Pocket Pedometer 793 & Waist & V \\
\hline Albrecht et al. (2020) & Accelerometer & ActiGraph wGT3X-BT & Wrist & V \\
\hline Al-Mohannadi et al. (2016) & Pedometer & Omron HJ-720 ITC & NA & V \\
\hline Arnardottir et al. (2017) & Accelerometer & ActiGraph GT3X+ & Hip & $S B, L P A, M V P A, V$ \\
\hline Aspvik et al. (2018) & Accelerometer & ActiGraph GT3X+ & Waist & V \\
\hline Atkin et al. (2016) & Accelerometer & ActiGraph GT1M & Waist & SB, MVPA \\
\hline Badland et al. (2011) & Pedometer & Yamax Digiwalker SW-200 & Hip & V \\
\hline Balish et al. (2017) & Pedometer & Yamax Digiwalker SW-200 & Hip & V \\
\hline $\begin{array}{l}\text { Barkley and Herrmann } \\
\text { (2017) }\end{array}$ & Accelerometer & ActiGraph GT3X & Waist & LPA, MVPA \\
\hline Beighle et al. (2013) & Pedometer & MLS 205 & Waist & V \\
\hline Bejarano et al. (2019) & Accelerometer & ActiGraph wActi Sleep-BT & Wrist & SB, MVPA \\
\hline Boutou et al. (2019) & Accelerometer & ActiGraph GT3X & Hip & MVPA, V \\
\hline Brandon et al. (2009) & Accelerometer & ActiGraph GT1M & Torso & V \\
\hline Bremer et al. (2019) & Pedometer & PiezoRX & Hip & V \\
\hline Bringolf-Isler et al. (2009) & Accelerometer & ActiGraph AM7164 & Waist & V \\
\hline Brychta et al. (2016) & Accelerometer & ActiGraph GT3X & Hip & V \\
\hline Buchowski et al. (2009) & Accelerometer & Tritrac-R3D & NA & $S B, V$ \\
\hline Button et al. (2020) & Accelerometer & Actical Z & Hip & LPA, MVPA \\
\hline Carr et al. (2016) & Pedometer & Omron HJ-720 ITC & NA & V \\
\hline Cepeda et al. (2018) & Accelerometer & GENEActiv & Wrist & SB, LPA, MVPA \\
\hline Chang et al. (2020) & Accelerometer & ActiGraph GT3X & Hip & $S B, L P A, M V P A, V$ \\
\hline Clemes et al. (2011) & Pedometer & Yamax Digiwalker SW-200 & Waist & V \\
\hline Collings et al. (2020) & Accelerometer & ActiGraph GT3X+ & Hip & $S B, L P A, M V P A, V$ \\
\hline Colom et al. (2019) & Accelerometer & GENEActiv & Wrist & MVPA \\
\hline Cooper et al. (2010) & Accelerometer & ActiGraph GT1M & Waist & V \\
\hline Cradock et al. (2009) & Accelerometer & TriTac-R3D & Hip & MVPA \\
\hline Crowley et al. (2016) & Pedometer & Jawbone UP & Wrist & V \\
\hline Cullen et al. (2017) & Accelerometer & ActiGraph GT3X+ & NA & V \\
\hline Davis et al. (2011) & Accelerometer & ActiGraph GT1M & Waist & LPA \\
\hline de Vries et al. (2019) & Accelerometer & 1. ActiWatch 2; 2. GENEActiv Original; 3. Sensewear Mini & $\begin{array}{l}\text { 1. Wrist; 2. Wrist; } 3 . \\
\text { Arm }\end{array}$ & SB, LPA, MVPA, V \\
\hline Declòs-Alió et al. (2019) & Accelerometer & ActiGraph GT3X & NA & V \\
\hline Deng and Fredriksen (2018) & Accelerometer & ActiGraph wGT3X-BT & Hip & MVPA \\
\hline Dias et al. (2019) & Accelerometer & 1. ActiGraph 7164; 2. ActiGraph GT1M; 3. ActiGraph 71256 & Waist & $S B, V, M V P A$ \\
\hline Diaz et al. (2016) & Accelerometer & Actical & Waist & SB \\
\hline Dill et al. (2014) & Accelerometer & ActiGraph GT3X & Hip & V, MVPA \\
\hline Duncan et al. (2008) & Pedometer & New Lifestyles 2000 & Waist & V \\
\hline Edwards et al. (2015) & Accelerometer & RT3 & Hip & $S B, M V P A, V$ \\
\hline Feinglass et al. (2011) & Accelerometer & ActiGraph GT1M & Hip & V \\
\hline Goodman et al. (2012) & Accelerometer & RT3 & Waist & MVPA \\
\hline
\end{tabular}


Table 2 Characteristics of devices used to measure physical activity and sedentary behavior (Continued)

\begin{tabular}{|c|c|c|c|c|}
\hline Reference & $\begin{array}{l}\text { Type of } \\
\text { Device }\end{array}$ & Specific Device & Wear Location & $\begin{array}{l}\text { Measure(s) of } \\
\text { Activity }\end{array}$ \\
\hline Goodman et al. (2014) & Accelerometer & 1. ActiGraph 7164; 2. ActiGraph GT1M; 3. ActiGraph 71256 & Waist & V \\
\hline Gracia-Marco et al. (2013) & Accelerometer & ActiGraph GT1M & Back & SB, MVPA, V \\
\hline Griew et al. (2010) & Accelerometer & ActiGraph GT1M & Hiр & V \\
\hline Hagströmer et al. (2014) & Accelerometer & ActiGraph 7164 & Hip & SB, MVPA, V \\
\hline Hamilton et al. (2009) & Pedometer & New Lifestyles Digi-Walker SW-200 & NA & V \\
\hline Harrison et al. (2011) & Accelerometer & ActiGraph GT1M & Hip & $S B, V, M V P A$ \\
\hline Harrison et al. (2015) & Accelerometer & ActiGraph GT1M & Hip & $S B, M V P A, V$ \\
\hline Harrison et al. (2017) & Accelerometer & 1. ActiGraph 7164; 2. ActiGraph GT1M & NA & V \\
\hline Hjorth et al. (2013) & Accelerometer & ActiGraph GT3X+ & Hip & $S B, M V P A, V$ \\
\hline Hoaas et al. (2019) & Accelerometer & SenseWear Pro & Arm & SB, LPA, MVPA, V \\
\hline Hopkins et al. (2011) & Accelerometer & ActiGraph GT1M & Hip & V \\
\hline Hoppmann et al. (2017) & Accelerometer & ActiGraph GT3X & Hip & MVPA, V \\
\hline Hunter et al. (2019) & Accelerometer & ActiGraph wGT3X-BT & Waist & SB, LPA, MVPA, V \\
\hline Jehn et al. (2014) & Accelerometer & AiperMotion & Hip & V \\
\hline Jones et al. (2017) & Accelerometer & ActiGraph GT1M & Waist & V \\
\hline Jones et al. (2017) & Accelerometer & ActiGraph GT1M & Waist & V \\
\hline Katapally et al. (2016) & Accelerometer & Actical & Waist & SB \\
\hline Kharlova et al. (2020) & Accelerometer & ActiGraph wGT3X-BT & Hip & SB, MVPA \\
\hline Kimura et al. (2015) & Pedometer & Kenz Lifecorder EX & Waist & V \\
\hline King et al. (2011) & Accelerometer & 1. ActiGraph 7164; 2. ActiGraph GT1M & Hip & $S B, V$ \\
\hline Kolle et al. (2009) & Accelerometer & ActiGraph MTI 7164 & Waist & V \\
\hline Kong et al. (2020) & Accelerometer & Fitbit Flex & Wrist & MVPA, V \\
\hline Koolhaas et al. (2017) & Accelerometer & GENEActiv & Wrist & SB, LPA, MVPA \\
\hline Larouche et al. (2019) & Pedometer & SC-Step Rx & NA & MVPA, V \\
\hline Lewis et al. (2016) & Accelerometer & ActiGraph GT3X+ & Hip & SB, MVPA \\
\hline Ma et al. (2018) & Accelerometer & 1. iPhones 55; 2. iPhone 6 & NA & V \\
\hline Martins et al. (2015) & Accelerometer & ActiGraph GT3X+ & Hip & MVPA \\
\hline McCrorie et al. (2015) & Accelerometer & activPAL & Thigh & V \\
\hline Mckee et al. (2012) & Pedometer & DigiWalker DW-2000 & NA & V \\
\hline McMurdo et al. (2012) & Accelerometer & RT3 & Hip & V \\
\hline Mitchell et al. (2018) & Accelerometer & Actical & NA & V \\
\hline Mitsui et al. (2010) & Pedometer & Yamasa EM-180 & NA & V \\
\hline Nagy et al. (2019) & Accelerometer & ActiGraph GT3X+ & Hip & SB, MVPA, V \\
\hline Nakashima et al. (2019) & Accelerometer & LifeLyzer05 Coach & Back & LPA, MVPA, V \\
\hline Newman et al. (2009) & Pedometer & Accusplit AE120 & Hip & V \\
\hline Nilsen et al. (2019) & Accelerometer & ActiGraph GT3X+ & Hip & SB, LPA, MVPA, V \\
\hline O'Connell et al. (2013) & Accelerometer & ActiGraph GT1M & Hip & SB, LPA, MVPA \\
\hline Ogawa et al. (2018) & Accelerometer & Kenz Lifecorder GS & Waist & MVPA, V \\
\hline Oliver et al. (2011) & Accelerometer & Mini-mitter & NA & MVPA \\
\hline Pagels et al. (2016) & Accelerometer & ActiGraph GT3X+ & NA & MVPA \\
\hline Patnode et al. (2010) & Accelerometer & ActiGraph 7164 & Hip & MVPA \\
\hline Pearce et al. (2012) & Accelerometer & ActiGraph GT1M & Hip & V \\
\hline Pechova et al. (2019) & Accelerometer & ActiGraph GT1M & Hip & SB \\
\hline
\end{tabular}


Table 2 Characteristics of devices used to measure physical activity and sedentary behavior (Continued)

\begin{tabular}{|c|c|c|c|c|}
\hline Reference & $\begin{array}{l}\text { Type of } \\
\text { Device }\end{array}$ & Specific Device & Wear Location & $\begin{array}{l}\text { Measure(s) of } \\
\text { Activity }\end{array}$ \\
\hline Pelclova et al. (2010) & Pedometer & Omron HJ-105 & Hip & V \\
\hline Prins and van Lenthe (2015) & GPS Logger & QStarz BT-Q1000XT & Hip & V \\
\hline Rahman et al. (2019) & Pedometer & Omron HJ-720 ITC & Hip & V \\
\hline Remmers et al. (2017) & Accelerometer & ActiGraph GT3X+ & Hip & SB, LPA, MVPA \\
\hline Ridgers et al. (2015) & Accelerometer & ActiGraph GT3X+ & Hip & MVPA \\
\hline Ridgers et al. (2018) & Accelerometer & ActiGraph GT3X+ & Hip & LPA, MVPA \\
\hline Robbins et al. (2013) & Accelerometer & ActiGraph GT1M & Hip & MVPA, V \\
\hline Rosenthal et al. (2020) & Accelerometer & NA & NA & V \\
\hline Rowlands et al. (2009) & Accelerometer & ActiGraph GT1M & Hip & MVPA, V \\
\hline Sartini et al. (2017) & Accelerometer & ActiGraph GT3X & Hip & SB, LPA, MVPA, V \\
\hline Schepps et al. (2018) & Accelerometer & ActiGraph GT3X+ & Hip & SB, LPA, MVPA, V \\
\hline Sewell et al. (2010) & Accelerometer & Gaehwiler Electronic Z80-32 kV1 & Waist & V \\
\hline Shen et al. (2013) & Accelerometer & ActiGraph GT1M & Hip & LPA, MVPA \\
\hline Silva et al. (2011) & Accelerometer & ActiGraph MTI/CSA 7164 & Hip & SB, MVPA \\
\hline Sit et al. (2019) & Accelerometer & ActiGraph GT3X & Hip & SB, MVPA \\
\hline Stabell et al. (2020) & Pedometer & Omron HJ-324 U & Waist & V \\
\hline Sugino et al. (2012) & Accelerometer & 1. Actimarker; 2. Dynaport Activity Monitor (DAM) & Waist & V \\
\hline Sumukadas et al. (2008) & Accelerometer & RT3 & Hip & V \\
\hline Van Kann et al. (2016) & Accelerometer & Actiheart & Chest & LPA, MVPA \\
\hline Wang et al. (2017) & Accelerometer & ActiGraph GT3X & Waist & MVPA, V \\
\hline Witham et al. (2014) & Accelerometer & RT3 & Waist & V \\
\hline Wong et al. (2020) & Accelerometer & ActiGraph GT3X+ & Hip & SB \\
\hline Wu et al. (2017) & Accelerometer & ActiGraph GT1M & Wrist & $S B, V$ \\
\hline Yildrim et al. (2012) & Accelerometer & $\begin{array}{l}\text { 1. ActiGraph ActiTrainer; 2. ActiGraph GT1M; 3. ActiGraph } \\
\text { GT3X }\end{array}$ & Waist & SB, LPA, MVPA, V \\
\hline Zheng et al. (2019) & Accelerometer & activPAL & Thigh & SB, MVPA \\
\hline
\end{tabular}

Note. $V$ Physical activity volume, LPA light-intensity physical activity duration, MVPA moderate-to-vigorous intensity physical activity duration, SB sedentary behavior duration

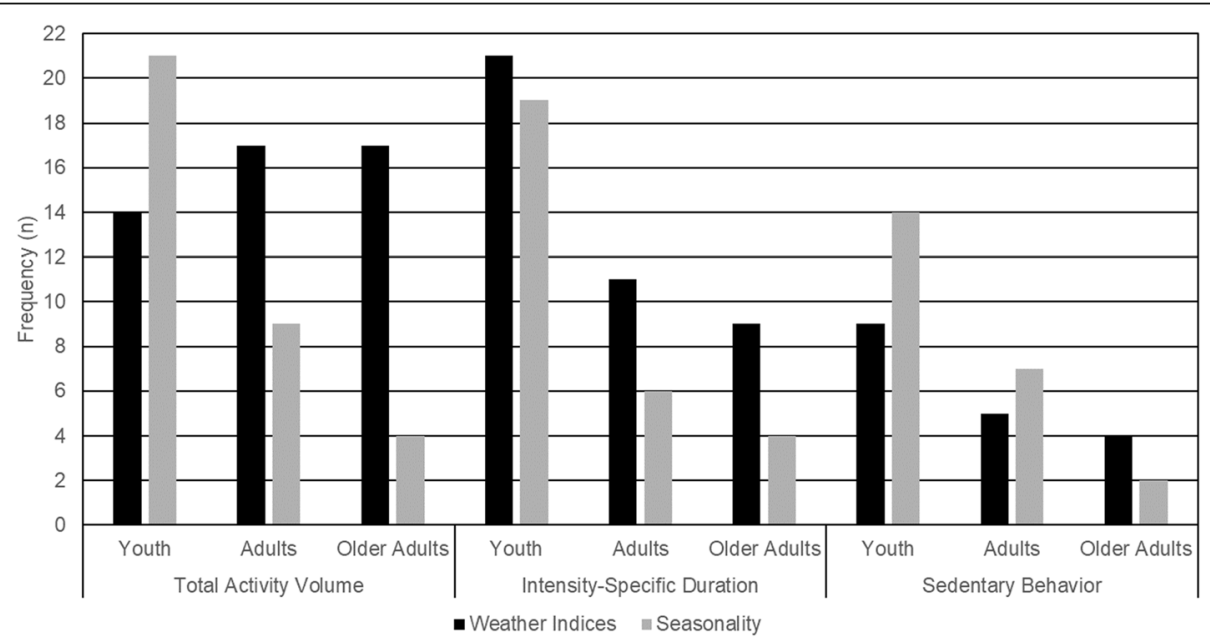

Fig. 3 Movement behavior measures in studies examining seasonality and weather indices across the lifespan 
Table 3 Seasonal differences in movement-related behaviors

\begin{tabular}{|c|c|c|c|c|c|}
\hline Season & $\begin{array}{l}\text { Reference } \\
\text { Season }\end{array}$ & Physical Activity Volume & $\begin{array}{l}\text { Light-Intensity } \\
\text { Physical Activity }\end{array}$ & $\begin{array}{l}\text { Moderate-to-Vigorous Intensity } \\
\text { Physical Activity }\end{array}$ & Sedentary Behavior \\
\hline \multirow[t]{12}{*}{ Winter } & $>$ Summer & $1[82]$ & 0 & $1[79]$ & $\begin{array}{l}10[30,47,51,52,58,67, \\
72,74,75,79]\end{array}$ \\
\hline & $=$ Summer & $6[65,72,74,80,83,84]$ & $6[52,55,79,80,85]$ & $7[65,67,74,80,85]$ & $3[65,74,80]$ \\
\hline & $<$ Summer & $\begin{array}{l}23[28,31,44,52,74-77,86- \\
99]\end{array}$ & $6[53,67,74,89,100]$ & $11[31,51-53,58,72,89,100-102]$ & 0 \\
\hline & $\begin{array}{l}\text { Evidence } \\
\text { Grade }\end{array}$ & Strong & Moderate & Strong & Strong \\
\hline & > Spring & 0 & 0 & 0 & $\begin{array}{l}11[51,52,54,55,67-69, \\
72,74,79]\end{array}$ \\
\hline & $=$ Spring & $7[29,49,65,68,80,84,98]$ & $7[30,52,74,80,100]$ & $10[49,53,55,65,67,68,79,80]$ & $\begin{array}{l}5 \\
{[53,65,68,77,80]}\end{array}$ \\
\hline & $<$ Spring & $\begin{array}{l}15[29,52,54,68,72,74,89 \\
90,103-107]\end{array}$ & $7[53-55,64,67,79,89]$ & $\begin{array}{l}12[33,52,54,68,72,74,89,100,101, \\
105]\end{array}$ & 0 \\
\hline & $\begin{array}{l}\text { Evidence } \\
\text { Grade }\end{array}$ & Strong & Moderate & Strong & Strong \\
\hline & $>$ Autumn & 0 & $2[79,100]$ & $1[79]$ & $3[52,67,74]$ \\
\hline & $=$ Autumn & $7[65,68,72,74,80,84,98]$ & $4[52,55,74,80]$ & $12[49,52,65-68,72,74,80,101]$ & $\begin{array}{l}8[65,66,68,72,74,79, \\
80]\end{array}$ \\
\hline & $<$ Autumn & $5[40,52,66,74,104]$ & $5[32,66,67,74]$ & $4[32,40,100]$ & 0 \\
\hline & $\begin{array}{l}\text { Evidence } \\
\text { Grade }\end{array}$ & Moderate & Limited & Strong & Moderate \\
\hline \multirow[t]{8}{*}{ Spring } & $>$ Autumn & $5[29,52,65,90]$ & $1[67]$ & $5[33,52,65,79]$ & 0 \\
\hline & $=$ Autumn & $6[29,49,80,84,98,105]$ & $5[30,52,64,79,80]$ & $7[30,49,55,67,80,105]$ & $5[53,55,65,79,80]$ \\
\hline & $<$ Autumn & $1[104]$ & $1[30]$ & 0 & $4[52,63,67,77]$ \\
\hline & $\begin{array}{l}\text { Evidence } \\
\text { Grade }\end{array}$ & Moderate & Limited & Moderate & Limited \\
\hline & $>$ Summer & $2[52,82]$ & $1[79]$ & $2[33,79]$ & 0 \\
\hline & $=$ Summer & $6[65,80,84,98,105,108]$ & $7[30,52,64,67,80,108]$ & $10[30,49,52,55,65,67,80,105,108]$ & $\begin{array}{l}8[53,55,65,67,69,79 \\
80,108]\end{array}$ \\
\hline & $<$ Summer & $2[87,104]$ & 0 & $1[41]$ & $2[52,77]$ \\
\hline & $\begin{array}{l}\text { Evidence } \\
\text { Grade }\end{array}$ & Limited & Moderate & Strong & Moderate \\
\hline \multirow[t]{4}{*}{ Summer } & $>$ Autumn & $1[87]$ & $1[67]$ & 0 & 0 \\
\hline & $=$ Autumn & $5[52,65,80,84,98]$ & $2[52,79]$ & $5[52,65,67,79,80]$ & $4[52,65,69,80]$ \\
\hline & $<$ Autumn & $1[82]$ & 0 & 0 & $2[67,79]$ \\
\hline & $\begin{array}{l}\text { Evidence } \\
\text { Grade }\end{array}$ & Limited & Not Assignable & Moderate & Limited \\
\hline
\end{tabular}

Strong evidence indicated that physical activity volume was greater in summer than winter $(23 / 30,76.7 \%)$, although one study found it was greater in winter than summer $(1 / 30,3.3 \%)$. Six studies $(20.0 \%)$ comparing volume in winter and summer yielded null results. Strong evidence also indicated that physical activity volume was consistently greater in spring than winter $(15 / 22,68.2 \%)$, but seven studies indicated no difference (7/22, 31.8\%). No studies found evidence of greater volume in winter than spring.
Moderate evidence indicated mixed findings for physical activity volume between spring and autumn with most studies revealing either greater volume in spring than autumn $(5 / 12,41.7 \%)$ or no differences in volume $(6 / 12,50.0 \%)$. One study reported greater volume in autumn than spring $(1 / 12,8.3 \%)$. Moderate evidence indicated mixed findings for physical activity volume between autumn and winter with some studies indicating no difference $(7 / 12,58.3 \%)$ and others indicating great volume in autumn than winter (5/12, 41.7\%). No 
studies found evidence of greater physical activity volume in winter than autumn.

Limited evidence compared physical activity volume in spring and summer were equivocal: six studies indicated no difference in volume between summer and spring (6/ $10,60 \%)$ but the remaining studies were evenly split between greater volume in summer $(2 / 10,20.0 \%)$ and spring $(2 / 10,20.0 \%)$. Limited evidence compared physical activity volume between autumn and summer. Findings generally indicated no difference $(5 / 7,71.4 \%)$ with the exception of one study indicating greater volume in autumn than summer $(1 / 7,14.3 \%)$ and another study indicating greater volume in summer than autumn $(5 / 7$, $14.3 \%)$.

\section{Intensity-specific physical activity duration}

Seasonal comparisons more frequently involved the duration of MVPA (37/37, 100.0\%) than LPA (20/37, $54.1 \%)$. Studies comparing MVPA duration between seasons sampled youth $(25 / 37,67.6 \%)$, adults $(6 / 37,16.2 \%)$, and older adults $(6 / 67$ 16.7\%). Seasonal comparisons of LPA duration sampled youth (11/20, 55.0\%), older adults (6/20, 30.0\%), and adults (3/20, 15.0\%).

As shown in Table 3, the most common seasonal comparisons of intensity-specific behavior durations involved winter vs. spring (overall: 22/37 [59.5\%]; MVPA: 22/22 [100\%]; LPA: 14/22 [63.6\%]; winter vs. summer (overall: 19/37 [51.4\%]; MVPA: 19/19 [\%]; LPA: 12/19 [63.2\%]), winter vs. autumn (overall: 17/37 [45.9\%]; MVPA: 17/17 [100\%]; LPA: 11/17 [64.7\%]), spring vs. summer (overall: 13/37 [35.1\%]; MVPA: 13/13 [100\%]; LPA: 8/13 [61.5\%]), and spring vs. autumn (overall: 12/37 [32.4\%]; MVPA: 12/12 [100\%]; LPA: 7/12 [58.3\%]).

Strong evidence indicated either greater MVPA in spring than winter $(12 / 22,54.5 \%)$ or no difference between these seasons $(10 / 22,44.5 \%)$. No studies found greater MVPA duration in winter than spring. Based on moderate evidence, findings about LPA were mixed: half of the studies reported greater LPA in spring than winter $(7 / 14,50.0 \%)$ and half reported no difference in LPA between spring and winter $(7 / 14,50.0 \%)$. Strong evidence indicated that MVPA duration was generally greater in summer than winter $(11 / 19,57.9 \%)$. Six studies found no difference between these seasons $(6 / 19,20 \%)$ and one study found winter activity greater than summer $(1 / 30$, $3.3 \%)$. Based on moderate evidence, studies indicated either greater LPA in summer than winter $(6 / 12,50.0 \%)$ or no difference $(6 / 12,50.0 \%)$. Strong evidence found mixed findings for seasonal differences in MVPA between autumn and winter, either indicating no difference $(12 / 17,70.6 \%)$ or greater MVPA in autumn than winter $(4 / 17,23.5 \%)$. One study demonstrated greater MVPA duration in autumn compared to winter $(1 / 17,5.9 \%)$. Based on limited evidence, LPA was either greater in autumn than winter $(5 / 11,45.5 \%)$, not different between these seasons $(4 / 11,36.6 \%)$, or greater in winter than autumn $(2 / 11,18.2 \%)$. Strong evidence indicated that MVPA duration typically did not differ between summer and spring (10/13, 76.9\%), but two studies revealed greater MVPA durations in summer $(2 / 13,15.3 \%)$ and another revealed greater MVPA duration in spring (1/ 13, 7.7\%). Moderate evidence indicated that LPA generally did not differ between summer than spring $(7 / 8$, $87.5 \%)$ but one study indicated greater LPA in spring than summer $(1 / 8,12.5 \%)$.

Moderate evidence indicated mixed findings from comparisons of MVPA between spring and autumn, either indicating no differences $(7 / 12,58.3 \%)$ or greater MVPA in spring than autumn (5/12, 41.7\%). No study demonstrated greater MVPA duration in autumn compared to spring. Based on limited evidence, LPA generally did not differ between spring and autumn (5/7, $71.4 \%$ ); however, one study indicated greater LPA in spring than autumn $(1 / 7,14.3 \%)$ and another study indicated greater LPA in autumn than spring $(1 / 7,14.3 \%)$. Moderate evidence indicated that MVPA duration did not differ between summer and autumn in five studies $(5 / 5,100 \%)$. A grade was not assignable for LPA comparisons between summer and autumn.

\section{Sedentary behavior}

Studies comparing sedentary behavior between seasons sampled youth $(14 / 23,60.9 \%)$, adults $(7 / 23,30.4 \%)$ and older adults $(2 / 23,8.7 \%)$. The most common seasonal comparisons of volume involved winter vs. spring (16/ $23,69.6 \%)$, winter vs. summer $(13 / 23,56.5 \%)$, winter vs. autumn (11/23, 47.8\%), spring vs. summer (10/23, $43.5 \%)$, spring vs. autumn $(9 / 23,39.1 \%)$, and summer vs. autumn $(6 / 23,26.1 \%)$.

Strong evidence indicated that sedentary behavior was generally greater in winter than spring $(11 / 16,68.8 \%)$ although some studies indicated no difference $(5 / 16$, $31.3 \%)$. Strong evidence indicated that sedentary behavior was generally greater in winter than summer $(10 / 13$, $76.9 \%)$ with a few studies indicating no difference $(3 / 13$, 23.1\%). Moderate evidence indicated that sedentary behavior did not vary between autumn and winter (8/11, $72.7 \%$ ) but a few studies indicated greater sedentary behavior in winter than autumn (3/11, 27.3\%). Moderate evidence indicated that sedentary behavior generally did not differ between spring and summer $(8 / 10,80 \%)$ but two studied indicated greater sedentary behavior during summer $(2 / 10,20 \%)$. Limited evidence also indicated that sedentary behavior either did not differ between spring and autumn $(5 / 9,55.6 \%)$ or was greater in autumn than spring $(4 / 9,44.4 \%)$. Limited evidence indicated that sedentary behavior either did not differ 
Table 4 Associations between movement-related behaviors and specific weather indices

\begin{tabular}{|c|c|c|c|c|c|}
\hline $\begin{array}{l}\text { Weather } \\
\text { Index }\end{array}$ & Finding & Physical Activity Volume & $\begin{array}{l}\text { Light-Intensity } \\
\text { Physical Activity }\end{array}$ & $\begin{array}{l}\text { Moderate-to-Vigorous } \\
\text { Intensity Physical Activity }\end{array}$ & Sedentary Behavior \\
\hline \multirow[t]{4}{*}{ Temperature } & $\begin{array}{l}\text { Positive } \\
\text { Associations }\end{array}$ & $19[35-38,40,43,47,70,88,107-114]$ & $4[47,64,70,115]$ & $\begin{array}{l}11[35,39,42,47,57,73,79, \\
81,110,116]\end{array}$ & $2[38,81]$ \\
\hline & $\begin{array}{l}\text { Null } \\
\text { Associations }\end{array}$ & $13[28,35-37,61,117-122]$ & $4[34,56,116]$ & $\begin{array}{l}14[34,35,40,42,56,61,70 \\
120,121,123,124]\end{array}$ & $\begin{array}{l}7[34,63,70,73,81 \\
115]\end{array}$ \\
\hline & $\begin{array}{l}\text { Negative } \\
\text { Associations }\end{array}$ & $6[38,46,125-128]$ & 0 & $3[81,122,124]$ & $\begin{array}{l}9[38,39,47,60-63,71, \\
109]\end{array}$ \\
\hline & $\begin{array}{l}\text { Evidence } \\
\text { Grade }\end{array}$ & Strong & Limited & Strong & Moderate \\
\hline \multirow[t]{4}{*}{ Precipitation } & $\begin{array}{l}\text { Positive } \\
\text { Associations }\end{array}$ & $1[28]$ & 0 & $2[79,123]$ & $\begin{array}{l}12[38,39,48,60,61 \\
63,71,73,81,109,129]\end{array}$ \\
\hline & $\begin{array}{l}\text { Null } \\
\text { Associations }\end{array}$ & $5[37,70,114,118]$ & $3[34,56,70]$ & $\begin{array}{l}15[35,39,42,56,70,81,121, \\
122,124,130,131]\end{array}$ & $7[34,39,62,63,70,81]$ \\
\hline & $\begin{array}{l}\text { Negative } \\
\text { Associations }\end{array}$ & $\begin{array}{l}25[28,36-38,40,43,46,48,57,61,88,109 \\
111-113,119,121,122,125,127,131,132]\end{array}$ & $3[34,57,116]$ & $\begin{array}{l}15[34,40,48,57,61,73,81 \\
109,116,124,129,133,134]\end{array}$ & 0 \\
\hline & $\begin{array}{l}\text { Evidence } \\
\text { Grade }\end{array}$ & Strong & Limited & Strong & Strong \\
\hline \multirow[t]{4}{*}{ Wind Speed } & $\begin{array}{l}\text { Positive } \\
\text { Associations }\end{array}$ & 0 & 0 & $1[39]$ & $2[48,60]$ \\
\hline & $\begin{array}{l}\text { Null } \\
\text { Associations }\end{array}$ & $6[28,36,40,43]$ & $2[34]$ & $3[34,40]$ & $4[34,39]$ \\
\hline & $\begin{array}{l}\text { Negative } \\
\text { Associations }\end{array}$ & $6[36,46,48,109,113,127]$ & 0 & $3[39,48,135]$ & 0 \\
\hline & $\begin{array}{l}\text { Evidence } \\
\text { Grade }\end{array}$ & Moderate & Not Assignable & Limited & Limited \\
\hline \multirow[t]{4}{*}{ Photoperiod } & $\begin{array}{l}\text { Positive } \\
\text { Associations }\end{array}$ & $\begin{array}{l}15[38,46-48,50,72,78,88,109,114,125 \\
127,134,136,137]\end{array}$ & $4[34,47,64,78]$ & $\begin{array}{l}9[34,48,50,72,73,78,133, \\
135]\end{array}$ & $1[63]$ \\
\hline & $\begin{array}{l}\text { Null } \\
\text { Associations }\end{array}$ & $6[43,113,118,121,122]$ & $1[34]$ & $6[47,79,81,121,122]$ & $3[34,81]$ \\
\hline & $\begin{array}{l}\text { Negative } \\
\text { Associations }\end{array}$ & 0 & 0 & 0 & $\begin{array}{l}10[38,47,48,50,63 \\
71-73,78,81]\end{array}$ \\
\hline & $\begin{array}{l}\text { Evidence } \\
\text { Grade }\end{array}$ & Strong & Limited & Strong & Strong \\
\hline \multirow[t]{4}{*}{ Snow } & $\begin{array}{l}\text { Positive } \\
\text { Associations }\end{array}$ & 0 & 0 & 0 & 0 \\
\hline & $\begin{array}{l}\text { Null } \\
\text { Associations }\end{array}$ & $3[111,114,125]$ & $1[116]$ & $4[39,42,116]$ & 0 \\
\hline & $\begin{array}{l}\text { Negative } \\
\text { Associations }\end{array}$ & $4[38,88,93]$ & $1[64]$ & $1[40]$ & 0 \\
\hline & $\begin{array}{l}\text { Evidence } \\
\text { Grade }\end{array}$ & Limited & Not Assignable & Limited & Not Assignable \\
\hline \multirow[t]{4}{*}{$\begin{array}{l}\text { Cloud } \\
\text { Coverage }\end{array}$} & $\begin{array}{l}\text { Positive } \\
\text { Associations }\end{array}$ & $1[127]$ & 0 & 0 & 0 \\
\hline & $\begin{array}{l}\text { Null } \\
\text { Associations }\end{array}$ & $2[36,37]$ & 0 & 0 & 0 \\
\hline & $\begin{array}{l}\text { Negative } \\
\text { Associations }\end{array}$ & $2[36,37]$ & 0 & $2[41,135]$ & 0 \\
\hline & $\begin{array}{l}\text { Evidence } \\
\text { Grade }\end{array}$ & Limited & Not Assignable & Not Assignable & Not Assignable \\
\hline \multirow[t]{3}{*}{ Humidity } & $\begin{array}{l}\text { Positive } \\
\text { Associations }\end{array}$ & 0 & 0 & 0 & $2[78,81]$ \\
\hline & $\begin{array}{l}\text { Null } \\
\text { Associations }\end{array}$ & 0 & $1[34]$ & $6[39,81,124]$ & $5[34,39,81]$ \\
\hline & Negative & $5[78,107,126,127]$ & $2[34,78]$ & $6[34,78,79,81,124]$ & 0 \\
\hline
\end{tabular}


Table 4 Associations between movement-related behaviors and specific weather indices (Continued)

\begin{tabular}{|c|c|c|c|c|c|}
\hline $\begin{array}{l}\text { Weather } \\
\text { Index }\end{array}$ & Finding & Physical Activity Volume & $\begin{array}{l}\text { Light-Intensity } \\
\text { Physical Activity }\end{array}$ & $\begin{array}{l}\text { Moderate-to-Vigorous } \\
\text { Intensity Physical Activity }\end{array}$ & Sedentary Behavior \\
\hline & Associations & & & & \\
\hline & $\begin{array}{l}\text { Evidence } \\
\text { Grade }\end{array}$ & Moderate & Not Assignable & Moderate & Limited \\
\hline \multirow[t]{4}{*}{ Visibility } & $\begin{array}{l}\text { Positive } \\
\text { Associations }\end{array}$ & $1[73]$ & 0 & 0 & 0 \\
\hline & $\begin{array}{l}\text { Null } \\
\text { Associations }\end{array}$ & 0 & $1[34]$ & 0 & $2[34]$ \\
\hline & $\begin{array}{l}\text { Negative } \\
\text { Associations }\end{array}$ & 1 [127] & $1[34]$ & $1[34]$ & 0 \\
\hline & $\begin{array}{l}\text { Evidence } \\
\text { Grade }\end{array}$ & Not Assignable & Not Assignable & Not Assignable & Not Assignable \\
\hline \multirow[t]{4}{*}{$\begin{array}{l}\text { Barometric } \\
\text { Pressure }\end{array}$} & $\begin{array}{l}\text { Positive } \\
\text { Associations }\end{array}$ & $1[127]$ & 0 & 0 & 0 \\
\hline & $\begin{array}{l}\text { Null } \\
\text { Associations }\end{array}$ & 0 & 0 & 0 & 0 \\
\hline & $\begin{array}{l}\text { Negative } \\
\text { Associations }\end{array}$ & 0 & 0 & 0 & 0 \\
\hline & $\begin{array}{l}\text { Evidence } \\
\text { Grade }\end{array}$ & Not Assignable & Not Assignable & Not Assignable & Not Assignable \\
\hline \multirow[t]{4}{*}{ Wind Chill } & $\begin{array}{l}\text { Positive } \\
\text { Associations }\end{array}$ & $1[73]$ & 0 & 0 & 0 \\
\hline & $\begin{array}{l}\text { Null } \\
\text { Associations }\end{array}$ & 0 & 0 & 0 & 0 \\
\hline & $\begin{array}{l}\text { Negative } \\
\text { Associations }\end{array}$ & 0 & 0 & 0 & 0 \\
\hline & $\begin{array}{l}\text { Evidence } \\
\text { Grade }\end{array}$ & Not Assignable & Not Assignable & Not Assignable & Not Assignable \\
\hline \multirow[t]{4}{*}{ Air Quality } & $\begin{array}{l}\text { Positive } \\
\text { Associations }\end{array}$ & 0 & 0 & 0 & 0 \\
\hline & $\begin{array}{l}\text { Null } \\
\text { Associations }\end{array}$ & 1 [120] & 0 & $1[120]$ & 0 \\
\hline & $\begin{array}{l}\text { Negative } \\
\text { Associations }\end{array}$ & 1 [138] & 0 & 0 & 0 \\
\hline & $\begin{array}{l}\text { Evidence } \\
\text { Grade }\end{array}$ & Not Assignable & Not Assignable & Not Assignable & Not Assignable \\
\hline
\end{tabular}

between summer and autumn $(4 / 6,66.7 \%)$ or was greater in autumn than summer $(2 / 6,33.3 \%)$.

\section{Specific weather indices associated with movement- related behaviors}

Weather indices were typically collected from regional weather stations or national institutes (73/77, 94.8\%); few studies used self-reported weather indices (3/77, $3.9 \%$ ) or did not specify where weather indices were collected (1/77, 1.3\%). Associations between specific indices and behavior examined in five or more studies are discussed below; all available results are summarized in Table 4. The weather indices are defined in Appendix 2. The most frequently-reported weather indices included temperature $(60 / 77,77.9 \%)$, precipitation $(58 / 77,75.3 \%)$, photoperiod $(33 / 77,42.9 \%)$, wind speed $(20 / 77,26.0 \%)$, humidity $(16 / 77,20.8 \%)$, snow $(12 / 77,15.6 \%)$, and cloud coverage $(7 / 77,9.1 \%)$. No grade was assigned to research on barometric pressure, visibility, wind chill, or air quality due to insufficient evidence.

\section{Temperature}

Associations between temperature and movementrelated behaviors were estimated in youth (24/60, $40.0 \%)$, adults $(23 / 60,38.3 \%)$, and older adults $(13 / 60$, $21.7 \%)$. The most common behavioral measure in these studies was physical activity volume (38/60, $61.7 \%)$, followed by intensity-specific physical activity duration (overall: 30/60 [50.0\%]; MVPA: 28/30 [93.3\%]; LPA: 8/30 [26.7\%]) and sedentary behavior (18/60, 30.0\%). 
Associations between temperature and physical activity volume were more often positive $(19 / 38,50.0 \%)$ than null $(13 / 38,34.2 \%)$ or negative $(6 / 38,15.8 \%)$. No studies explicitly tested for curvilinear relations, but four studies noted an inverted-U pattern in which volume was lower during normatively warmer or colder days $[38,78,117$, 125]. Additional negative associations with volume were found when extreme temperatures were present [126, 127]. This evidence was graded as strong in favor of an inverted- $U$ relation between temperature and physical activity volume.

Moderate evidence indicated that associations between temperature and MVPA duration were more often null $(14 / 28,50.0 \%)$ or positive $(11 / 28,39.3 \%)$ than negative (3/28, 10.7\%). Limited evidence suggested that LPA exhibited a similar pattern with studies showing either a null $(4 / 8,50.0 \%)$ or positive $(4 / 8,50.0 \%)$ association with temperature. Moderate evidence indicated that sedentary behavior exhibited more negative associations with temperature $(9 / 18,50.0 \%)$ than null $(7 / 18,38.9 \%)$ or positive associations $(2 / 18,11.1 \%)$.

\section{Precipitation}

Associations between precipitation and movementrelated behaviors were estimated in youth $(28 / 58$, $48.3 \%)$, adults $(21 / 58,36.2 \%)$ and older adults $(9 / 58$, $15.5 \%)$. Precipitation was most frequently studied in relation with physical activity volume (31/58, 53.4\%) followed by intensity-specific physical activity durations (overall: 32/58 [55.2\%]; MVPA: 32/32 [100\%]; LPA: $6 / 32[18.8 \%]$ ) and sedentary behavior (19/32, $32.8 \%)$.

Strong evidence indicated that associations between precipitation and physical activity volume were largely negative $(25 / 31,80.6 \%)$ with a few null $(5 / 31,16.1 \%)$ and one positive $(1 / 31,3.2 \%)$ association. Strong evidence indicated that the association between precipitation and MVPA durations were mixed with negative $(15 / 32,46.9 \%)$ and null $(15 / 32,46.9 \%)$ results. Two studies reported a positive association between precipitation and MVPA duration (2/32, 6.3\%). Limited evidence indicated that precipitation and LPA had either a negative $(3 / 6,50.0 \%)$ or null $(3 / 6,50.0 \%)$ association. Moderate evidence indicated a mix of positive $(12 / 19,63.2 \%)$ and null associations $(7 / 19,36.8 \%)$ between precipitation and sedentary behavior. No studies reported a negative association between precipitation and sedentary behavior.

\section{Photoperiod}

Associations between the photoperiod and movementrelated behaviors were estimated in youth $(14 / 33$, $42.4 \%)$, adults $(10 / 33,30.3 \%)$ and older adults $(9 / 33$, 27.3\%). These studies reported associations between the photoperiod and measures of physical activity volume (21/33, 63.6\%), intensity-specific physical activity duration (overall: 19/33 [57.6\%]; MVPA: 15/19 [78.9\%], LPA: $5 / 19$ [26.3\%]) and sedentary behavior (14/33, 42.4\%).

Strong evidence indicated that associations between photoperiod and physical activity volume were mostly positive $(15 / 21,71.4 \%)$ with a few null associations $(6 / 21,28.6 \%)$. No studies reported a negative association between photoperiod and physical activity volume. Strong evidence indicated that associations between the photoperiod and MVPA duration were mostly positive $(9 / 15,60.0 \%)$ or null $(6 / 15,40.0 \%)$. No studies reported a negative association between photoperiod and MVPA duration. Limited evidence suggested a positive association between photoperiod and LPA duration $(4 / 5,80.0 \%)$ with a single study indicating a null association (1/5, 20.0\%). Strong evidence indicated that the photoperiod and sedentary behavior had a negative association (10/14, 71.4\%), with a few studies indicating either null $(3 / 14,21.4 \%)$ or positive associations (1/14, 7.1\%).

\section{Wind speed}

Associations between wind speed and movement-related behaviors were estimated in youth $(8 / 20,40.0 \%)$, older adults $(7 / 20,35.0 \%)$, and adults $(5 / 20,25.0 \%)$. These studies reported associations between wind speed and total physical activity volume $(12 / 20,60.0 \%)$ intensityspecific physical activity durations (overall: 7/20 [35.0\%]; MVPA: 7/7 [100\%]; LPA: 3/7 [42.9\%]), and sedentary behavior $(6 / 20,35.0 \%)$.

Moderate evidence indicated that associations between wind speed and physical activity volume were a mix of null (6/12, 50.0\%), and negative associations (6/12, $50.0 \%)$. No study reported a positive association between wind speed and volume.

Limited evidence indicated that wind speed and MVPA durations demonstrated mostly null (3/7, 42.9\%) or negative associations $(3 / 7,42.9 \%])$, although one study found a positive association $(1 / 7,14.3 \%)$. A grade was not assignable for evidence on wind speed and LPA. Limited evidence indicated that wind speed and sedentary behavior primarily displayed null $(4 / 6,66.7 \%)$ or positive associations $(2 / 6,33.3 \%)$; no studies reported a negative association.

\section{Humidity}

Associations between humidity and movement-related behaviors were estimated in adults $(7 / 16,43.8 \%)$, youth $(5 / 16,31.3 \%)$, and older adults $(4 / 16,25.0 \%)$. Studies including humidity frequently examined measures of physical activity volume (5/16, 31.3\%), followed in frequency by intensity-specific physical 
activity duration (overall: 12/16 [75.0\%]; MVPA: 12/12 [100\%]; LPA: 3/12 [25.0\%], sedentary behavior: 7/16 $[43.8 \%])$.

Moderate evidence indicated a consistently negative association between humidity and physical activity volume $(5 / 5,100.0 \%)$. Moderate evidence indicated a mix of negative $(6 / 12,50.0 \%)$ and null $(6 / 12,50.0 \%)$ associations between humidity and MVPA. A grade was not assignable to evidence linking humidity and LPA. Limited evidence suggested a null association between humidity and sedentary behavior $(5 / 7,71.4 \%)$ although some studies found a positive association (2/ 7, 28.6\%).

\section{Snow}

Associations between snow and movement-related behaviors were estimated in youth $(5 / 12,41.7 \%)$, adults (3/ $12,25.0 \%)$, and older adults $(4 / 12,33.3 \%)$. Studies including snow frequently examined measures of physical activity volume $(7 / 12,58.3 \%)$, followed in frequency by intensity-specific physical activity duration (overall: 6/12 [50.0\%]; MVPA: 5/6 [83.3\%], LPA: 2/6 [33.3\%]).

Limited evidence on snow and physical activity volume was split between negative $(4 / 7,57.1 \%)$ and null $(3 / 7$, $42.9 \%)$ associations. No study examining snow and volume demonstrated a positive association. Limited evidence on snow and MVPA duration was split between null $(4 / 5,80.0 \%)$ and negative $(1 / 5,20.0 \%)$ associations. A grade was not assignable to evidence linking snow with either LPA or sedentary behavior.

\section{Cloud coverage}

Associations between cloud coverage and movementrelated behaviors were estimated in youth $(4 / 7,57.1 \%)$, adults $(1 / 7,14.3 \%)$, and older adults $(2 / 7,28.6 \%)$. Studies including cloud coverage frequently examined measures of physical activity volume (5/7, 71.4\%), followed in frequency by intensity-specific physical activity duration (overall: 2/7 [28.6\%]; MVPA: 2/2 [100\%], LPA: 0/2 $[0.0 \%])$.

Limited evidence indicated that cloud coverage and physical activity volume exhibit a negative $(2 / 5,40.0 \%)$ or null associations $(2 / 5,40.0 \%)$. One study found a positive association between cloud coverage and physical activity volume $(1 / 5,40.0 \%)$. A grade was not assignable to evidence linking cloud coverage and durations of MVPA, LPA, or sedentary behavior.

\section{Discussion}

The present review summarized 144 studies from 110 articles with 118,189 participants from 30 countries. It updates conclusions from two seminal reviews based on a total of over 60 studies on over 300,000 participants from approximately 18 countries $[10,11]$. In those reviews, studies on seasonal differences in physical activity greatly outnumbered studies on weather correlates. Over the past decade, attention has been divided more equally between seasonal differences in and weather correlates of movement behaviors. This review also extended the prior reviews by capturing the spectrum of movement behaviors ranging from physical activity volume to intensityspecific durations to sedentary behavior. Collectively, these reviews establish how key features of the natural environment are linked with a variety of movement behaviors.

Physical activity volume and MVPA duration were the most frequent measures of movement behavior. Consistent with prior work, winter and summer were marked by the lowest and greatest movement behavior, respectively $[10,11]$. The present review extended those conclusions by documenting a trend in favor of greater physical activity volume and MVPA in spring than autumn. Comparisons of spring vs summer and summer vs autumn largely revealed no differences. Overall, the pattern of seasonal differences in physical activity volume and MVPA duration resembled a sinusoidal pattern and corresponded with both fluctuations in temperature and the waxing and waning photoperiod across the calendar year.

One contribution of the present review was that it examined a broad spectrum of movement behaviors that contribute to physical activity volume. MVPA duration has enjoyed a privileged status in the scientific literature because it has the strongest connections with health benefits [24]. MVPA also exhibited the clearest pattern of relations with seasonality and specific weather indices, including temperature, precipitation, and photoperiod. Thus, MVPA may provide one pathway by which the natural environment gets "under the skin" to affect health $[139,140]$.

Moving the needle on population-level MVPA has proven to be difficult [141, 142]. Far more time is spent in LPA than MVPA and LPA is a greater contributor to physical activity volume for most people [143]. Recent work has established unique health benefits from LPA after adjusting for MVPA [144]. As a consequence, LPA is a desirable substitute for prolonged sedentary behavior when MVPA is not feasible. Although a trend for increased LPA in summer and spring compared to winter was observed in the literature, LPA in the spring did not differ from LPA in the summer or autumn. In the interest of understanding how the natural environment facilitates or inhibits movement, this common form of physical activity should be a priority measure in future research examining seasonal differences and weather correlates. 
Sedentary behavior was consistently greater in winter than spring or summer. Weather has been cited as a barrier to physical activity that facilitates sedentary behavior $[8,9]$. This study extended previous work by reviewing how specific weather indices, as opposed to perceptions of the weather, are associated with sedentary behavior. People engage in more sedentary behavior on shorter days (photoperiod), when precipitation is greater, and when temperatures are lower. These findings revealed that weather is likely to be a third variable influencing the entire spectrum of movement-related behaviors.

These findings have two major implications. First, although the studies reviewed here were necessarily observational, they can inform behavioral interventions. Weather conditions that may serve as actual, as well as perceived, barriers to physical activity (e.g., too hot, cold, rainy or snowy, windy, or shorter days). Current or forecasted weather conditions may be useful for providing contextual information about opportunities for activity that could inform just-in-time interventions for movement behaviors. For example, users could be prompted to develop coping plans for exercise in adverse weather conditions and then reminded of those plans when adverse weather conditions were expected. Digital tools could also extend work on person-specific physical activity interventions by learning how to identify userspecific preferred weather patterns for movement behaviors and prompt users to ensure they capitalize on their preferred conditions to be active [145].

At a more general level, the impact of seasonal differences in movement behaviors on the implementation and evaluation of physical activity promotion programs should be considered when interpreting ambulatory behavior changes. Lifestyle physical activity intervention evaluations often last 1-6 months, and few last 12 months, so baseline and follow-up assessments are often conducted during different seasons. Seasonal influences on activity levels are typically allocated to the error term of statistical models but may be informative to include as a covariate or moderator of intervention effects. For example, including season as a moderator could reveal if interventions work better when days are lengthening (winter to summer) or when days are shortening (summer to winter).

Second, climate change is increasing the frequency of extreme weather conditions. Climatic zones that are currently favorable for movement behaviors may become inhospitable, inhibit physical activity, and contribute to health disparities. Some have speculated that climate change will increase migration as people seek to preserve an adaptive environmental niche [146]. Population-level data on movement behaviors could be investigated as a leading indicator of future health or migration due to climate change.
This review had limitations as well. The search was conducted using three databases and limited to English language publications. It is possible that studies were missed if they were published in journals not indexed by PubMed, CINAHL, or SPORTDiscus, or were written in languages other than English. All data were observational so strong causal inferences are not possible. Results were obtained from 30 countries on five continents but western and high-income countries were overrepresented in the data. Low- and middle-income countries are home to over $80 \%$ of the world's population and four times as many deaths are attributed to physical inactivity in those countries than in high-income countries [147, 148]. Conclusions may not generalize equally well to all regions, climatic zones, or economic strata. Most studies did not report on race or ethnicity so it is unclear how physical, social or cultural differences influence seasonal differences or relations between weather and movement behaviors. Devices were used to obtain measures of physical activity volume and intensity-specific duration - primarily MVPA - but some activity types do not lend themselves to accurate measurement with devices (e.g., cycling, swimming) or are sub-optimal with devices attached at the waist (e.g., sedentary behavior). Devices also provide no insights into the specific domains of physical activity (e.g., occupational, transport-related, occupational, domestic) or sedentary behavior (e.g., reading, screen time, socializing, eating). The available literature has focused almost exclusively on aggregated weather summaries during monitoring periods. Yet weather is dynamic so those summaries may not generalize to within-person change processes [149]. Additionally, physiological or psychological differences in environmental tolerances likely exist. Some people, for example, will be more heat tolerant or may simply enjoy running in the rain. Person-specific models of physical activity under different weather conditions could shed light on these dynamics [145]. Finally, the review focused on 11 common weather indices but other indices may also be relevant.

\section{Conclusions}

In sum, this review established consistent patterns of seasonal and weather-specific differences in physical activity and sedentary behavior. People tend to be most active in summer and least active in winter. This pattern coincides with temperature and photoperiod cycles. Weather may also influence comfort with physical activity as indicated by associations with precipitation and wind speed. These findings can inform physical activity promotion initiatives in the short-term and may have long-term implications for environmental influences on human health during the climate crisis. 


\section{Appendix A}

Table 5 Search Methods | PubMed

Search terms: ( ("Exercise"[MeSH] OR "Exercises"[tiab] OR

"Exercising"[MeSH] OR "Exercising"[tiab] OR "Physical activities"[MeSH] OR

"Physical activities"[tiab] OR "Physical activity"[MeSH] OR "Physical activity"[tiab] OR "Physical fitness"[MeSH] OR "Physical fitness"[tiab] OR "Recreation"[MeSH] OR "Recreation"[tiab] OR "Run"[MeSH] OR "Run"[tiab] OR "Running"[MeSH] OR "Running"[tiab] OR "Walk"[MeSH] OR "Walk"[tiab] OR "Walking"[MeSH] OR "Walking"[tiab]) OR ("Computer game"[MesH] OR "Computer game"[tiab] OR "Computer games"[MeSH] OR "Computer games"[tiab] OR "Computer usage"[MeSH] OR "Computer usage"[tiab] OR "Computer use"[MeSH] OR "Computer use"[tiab] OR "Physically inactive"[MeSH] OR "Physically inactive"[tiab] OR "Screen time"[MeSH] OR "Screen time"[tiab] OR "Sedentarism"[MeSH] OR "Sedentarism"[tiab] OR "Sedentary behavior"[MeSH] OR "Sedentary behavior" [tiab] OR "TV viewing"[MeSH] OR "TV viewing"[tiab] OR "TV watching"[MeSH] OR "TV watching"[tiab] OR "Video game"[MeSH] OR "Video game"[tiab] OR "Video games"[MeSH] OR "Video games"[tiab] OR "Video gaming"[MeSH] OR "Video gaming"[tiab])) AND

("Accelerometer"[MeSH] OR "Accelerometer"[tiab] OR

"Accelerometry"[MeSH] OR "Accelerometry"[tiab] OR "Pedometer"[MeSH] OR "Pedometer"[tiab] OR "Steps"[MeSH] OR "Steps"[tiab])

AND

(“"Season"[MeSH] OR "Season"[tiab] OR "Seasonal"[MeSH] OR

"Seasonal"[tiab] OR "Seasons"[MeSH] OR "Seasons"[tiab]) OR

("Atmospheric Pressure"[MesH] OR "Atmospheric Pressure"[tiab] OR

"Cloud"[MeSH] OR "Cloud"[tiab] OR "Cloud Cover"[MeSH] OR "Cloud

Cover"[tiab] OR "Clouds"[MeSH] OR "Clouds"[tiab] OR "Cloudy"[MeSH] OR

"Cloudy"[tiab] OR "Fog"[MeSH] OR "Fog"[tiab] OR "Heat index"[MeSH] OR

"Fog"[tiab] OR "Humid"[MeSH] OR "Humid"[tiab] OR "Humidity"[MeSH]

OR "Humidity"[tiab] OR "Ice"[MeSH] OR "Ice"[tiab] OR "Lightning"[MeSH]

OR "Lightning"[tiab] OR "Overcast"[MeSH] OR "Overcast"[tiab] OR "Precipitation"[MeSH] OR "Precipitation"[tiab] OR "Rain"[MeSH] OR "Rain"[tiab] OR "Saturation"[MeSH] OR "Saturation"[tiab] OR "Severe weather"[MeSH] OR "Severe weather" [tiab] OR "Snow"[MeSH] OR "Snow"[tiab] OR "Sunlight"[MeSH] OR "Sunlight"[tiab] OR "Temperature"[MeSH] OR "Temperature"[tiab] OR "Temperatures"[MeSH] OR "Temperatures"[tiab] OR "Ultraviolet rays"[MeSH] OR "Ultraviolet rays"[tiab] OR "UV index"[MeSH] OR "UV index"[tiab] OR "UV rays"[MeSH] OR "UV rays"[tiab] OR "Visibility"[MeSH] OR "Visibility"[tiab] OR "Weather"[MeSH] OR "Weather"[tiab] OR "Wind"[MeSH] OR "Wind"[tiab] OR "Wind chill"[MeSH] OR "Wind chill"[tiab] OR "Windy"[MeSH] OR "Windy"[tiab]))

\section{Limiters: (“2006/01/01"[Date - Publication]: “2020/10/31"[Date - Publication]) AND (English[Language])}

Filters: Humans

\section{Appendix B}

Table 6 Search Methods | CINAHL

Search terms: (MH "Exercise") OR (MH "Physical Fitness") OR (MH "Cardiorespiratory Fitness") OR (MH "Physical Activity") OR (MH "Recreation") OR (MH "Running, Distance") OR (MH "Running") OR (MH "Walking") OR (MH "Life Style, Sedentary") OR (MH "Television") OR (MH "Video Games") OR (MH "Computers, Portable") OR (MH "Computers, Hand-Held") OR (MH "Screen Time")OR TI "Exercise" OR "Physical Fitness" OR "Cardiorespiratory Fitness" OR "Physical Activity" OR "Recreation" OR "Running, Distance" OR "Running" OR "Walking" OR "Life Style, Sedentary" OR "Television" OR "Video Games" OR "Computers, Portable" OR "Computers, Hand-Held" OR "Screen Time" OR AB "Exercise" OR "Physical Fitness" OR "Cardiorespiratory Fitness" OR "Physical Activity" OR "Recreation" OR "Running, Distance" OR "Running" OR "Walking" OR "Life Style, Sedentary" OR "Television" OR "Video Games" OR "Computers, Portable" OR "Computers, Hand-Held" OR "Screen Time"

AND

(MH "Accelerometers") OR (MH "Pedometers") OR (MH "Step") OR (MH "Fitness Trackers") OR TI "Accelerometers" OR "Pedometers" OR "Step" OR "Fitness Trackers" OR "Wearable Electronic Devices" OR AB

"Accelerometers" OR "Pedometers" OR "Step" OR "Fitness Trackers" OR "Wearable Electronic Devices"

AND

(MH "Weather") OR (MH "Extreme Weather") OR (MH "Seasons") OR (MH "Temperature") OR (MH "Humidity") OR (MH "Heat") OR (MH "Ice") OR (MH "Ultraviolet Rays") OR (MH "Sunlight") OR (MH "Smog") OR (MH "Light") OR (MH "Lightning") OR (MH "Atmospheric Pressure") OR (MH "Rain") OR (MH "Snow") OR (MH "Atmosphere") OR (MH "Meteorological Factors") OR (MH "Climate") OR TI "Weather" OR "Extreme Weather" OR "Seasons" OR "Temperature" OR "Humidity" OR "Heat" OR "Ice" OR "Ultraviolet Rays" OR "Sunlight" OR "Smog" OR "Light" OR "Lightning" OR "Atmospheric Pressure" OR "Rain" OR "Snow" OR "Atmosphere" OR "Meteorological Factors" OR "Climate" OR AB "Weather" OR "Extreme Weather" OR "Seasons" OR "Temperature" OR "Humidity" OR "Heat" OR "Ice" OR "Ultraviolet Rays" OR "Sunlight" OR "Smog" OR "Light" OR "Lightning" OR "Atmospheric Pressure" OR "Rain" OR "Snow" OR "Atmosphere" OR "Meteorological Factors" OR "Climate"

Limiters: (“2006/01/01”[Date - Publication]: “2020/10/31”[Date Publication]) AND (English[Language])

Filters: Humans 


\section{Appendix C}

Table 7 Search Methods | SPORTDiscus

Search terms: (DE "EXERCISE") OR (DE "PHYSICAL fitness") OR (DE "PHYSICAL activity") OR (DE "RECREATION") OR (DE "RUNNING") OR (DE "WALKING") OR (DE "SEDENTARY behavior") OR (DE "SUBSCRIPTION television") OR (DE "VIDEO games") OR (DE "COMPUTER games") OR (DE "SEDENTARY people") OR TI ("EXERCISE") OR ("PHYSICAL fitness") OR ("PHYSICAL activity") OR ("RECREATION") OR ("RUNNING") OR ("WALKING") OR ("SEDENTARY behavior") OR ("SUBSCRIPTION television") OR ("VIDEO games") OR ("COMPUTER games") OR ("SEDENTARY people") OR AB ("EXERCISE") OR ("PHYSICAL fitness") OR ("PHYSICAL activity") OR ("RECREATION") OR ("RUNNING") OR ("WALKING") OR ("SEDENTARY behavior") OR ("SUBSCRIPTION television") OR ("VIDEO games") OR ("COMPUTER games") OR ("SEDENTARY people")

AND

(DE "PEDOMETERS") OR (DE "ACCELEROMETERS") OR ("PEDOMETERS") OR

("ACCELEROMETERS") OR ("STEP") OR ("FITNESS TRACKERS") OR ("WEARABLE ELEC

TRONIC DEVICES") OR AB ("PEDOMETERS") OR ("ACCELEROMETERS") OR ("STEP") OR ("FITNESS TRACKERS") OR ("WEARABLE ELECTRONIC DEVICES")

AND

(DE "WEATHER") OR (DE "TEMPERATURE") OR (DE "HUMIDITY") OR (DE "ICE") OR (DE "ULTRAVIOLET radiation") OR (DE "SUNSHINE") OR (DE "ATMOSPHERIC pressure") OR (DE "SNOW") OR (DE "COLD (Temperature)") OR TI ("WEATHER") OR ("TEMPERATURE") OR ("HUMIDITY") OR ("ICE") OR ("ULTRAVIOLET radiation") OR ("SUNSHINE") OR ("ATMOSPHERIC pressure") OR ("SNOW") OR ("COLD (Temperature)") OR ("ATMOSPHERE") OR ("METEOROLOGICAL FACTORS") OR ("EXTREME WEATHER") OR ("CLIMATE") OR AB ("WEATHER") OR ("TEMPERATURE") OR ("HUMIDITY") OR ("ICE") OR ("ULTRAVIOLET radiation") OR ("SUNSHINE") OR ("ATMOSPHERIC pressure") OR ("SNOW") OR ("COLD (Temperature)") OR ("ATMOSPHERE") OR ("METEOROLOGICAL FACTORS") OR ("EXTREME WEATHER") OR ("CLIMATE")

Limiters: ("2006/01/01"[Date - Publication]: "2020/10/31"[Date - Publication]) AND (English[Language])

Filters: Humans

\section{Appendix D}

Table 8 Glossary of Weather Indices

\begin{tabular}{|c|c|}
\hline Index & Definition \\
\hline Temperature & Degree or intensity of heat in the environment \\
\hline Precipitation & $\begin{array}{l}\text { Product of condensation from atmospheric water } \\
\text { vapor (typically rain and sleet in this review) }\end{array}$ \\
\hline Wind Speed & $\begin{array}{l}\text { Atmospheric quantity resulting from air traveling from } \\
\text { high to low pressure }\end{array}$ \\
\hline Photoperiod & Day length, or the period of daily illumination \\
\hline Snow & $\begin{array}{l}\text { Small, frozen white ice crystals generated from } \\
\text { atmospheric water vapor at temperatures below the } \\
\text { freezing point }\end{array}$ \\
\hline $\begin{array}{l}\text { Cloud } \\
\text { Coverage }\end{array}$ & $\begin{array}{l}\text { The fraction of the sky obscured by clouds in a } \\
\text { particular location }\end{array}$ \\
\hline Humidity & $\begin{array}{l}\text { Concentration of atmospheric water vapor present in } \\
\text { the environment }\end{array}$ \\
\hline Visibility & $\begin{array}{l}\text { Distance that can be seen due to light and weather } \\
\text { conditions }\end{array}$ \\
\hline $\begin{array}{l}\text { Barometric } \\
\text { Pressure }\end{array}$ & Pressure within the atmosphere \\
\hline Windchill & $\begin{array}{l}\text { Quantity of air temperature that is effectively lowered } \\
\text { due to wind }\end{array}$ \\
\hline Air Quality & A measure of how clean or polluted the air is \\
\hline
\end{tabular}

Note. Definitions obtained from the National Weather Service and the Oxford English Dictionary [150, 151]

\section{Supplementary Information}

The online version contains supplementary material available at https://doi. org/10.1186/s12966-021-01091-1.

\section{Additional file 1}

\section{Acknowledgements}

Not applicable.

\section{Authors' contributions}

ABW, SKM, CML, and DEC made substantial contributions to the conception or design of the work; TBT, KMB, SahH, SarH made substantial contributions to the acquisition, analysis and interpretation of data for the work. All authors contributed to drafting or revising the work critically for important intellectual content, approved the final version to be published, and agree to be accountable for all aspects of the work in ensuring that questions related to the accuracy or integrity of any part of the work are appropriately investigated and resolved.

\section{Funding}

Research reported in this publication was supported in part by the National Heart, Lung, and Blood Institute of the National Institutes of Health under award number R01HL142732 and the Division of Electrical, Communications, and Cyber Systems of the National Science Foundation under award number 1808266. Scherezade Mama is supported in part by a National Cancer Institute career development award (K07 CA222335). The content is solely the responsibility of the authors and does not necessarily represent the official views of the National Institutes of Health or the National Science Foundation. The funders had no role in the design of the study and collection, analysis, and interpretation of data and in writing the manuscript should be declared.

Availability of data and materials

All data generated or analyzed during this study are included in this published article and its supplementary information files.

\section{Ethics approval and consent to participate}

Not applicable.

\section{Consent for publication}

Not applicable.

\section{Competing interests}

The authors declare that they have no competing interests.

\section{Author details}

'Department of Kinesiology, The Pennsylvania State University, University Park, PA 16802, USA. ${ }^{2}$ Advanced Safety \& User Experience, Aptiv, Troy, MI, USA. ${ }^{3}$ Department of Electrical Engineering \& Computer Science, The Pennsylvania State University, University Park, PA, USA. ${ }^{4}$ Department of Health Disparities Research, The University of Texas MD Anderson Cancer Center, Houston, TX, USA. ${ }^{5}$ Department of Preventive Medicine, Northwestern University, Chicago, IL, USA.

Received: 17 June 2020 Accepted: 22 January 2021

Published online: 04 February 2021

\section{References}

1. Guthold R, Stevens GA, Riley LM, Bull FC. Worldwide trends in insufficient physical activity from 2001 to 2016: a pooled analysis of 358 populationbased surveys with 1.9 million participants. Lancet Glob Health. 2018;6(10): e1077-86.

2. McLeroy KR, Bibeau D, Steckler A, Glanz K. An ecological perspective on health promotion programs. Health Educ Q. 1988;15(4):351-77. 
3. Sallis JF, Cervero RB, Ascher W, Henderson KA, Kraft MK, Kerr J. An ecological approach to creating active living communities. Annu Rev Public Health. 2006;27:297-322.

4. Spence JC, Lee RE. Toward a comprehensive model of physical activity. Psychol Sport Exerc. 2003;4(1):7-24.

5. Bopp M, Gayah W, Campbell ME. Examining the link between public transit use and active commuting. Int J Environ Res Public Health. 2015 Apr;12(4): 4256-74.

6. Burton NW, Turrell G, Oldenburg B. Participation in recreational physical activity: why do socioeconomic groups differ? Health Educ Behav. 2003. 30(2):225-44.

7. Tappe MK, Duda JL, Ehrnwald PM. Perceived barriers to exercise among adolescents. J Sch Health. 1989 Apr 1;59(4):153-5.

8. Salmon J, Owen N, Crawford D, Bauman A, Sallis JF. Physical activity and sedentary behavior: a population-based study of barriers, enjoyment, and preference. Health Psychol. 2003;22(2):178-88.

9. O'Donoghue G, Perchoux C, Mensah K, Lakerveld J, van der Ploeg H, Bernaards $C$, et al. A systematic review of correlates of sedentary behaviour in adults aged 18-65 years: a socio-ecological approach. BMC Public Health. 2016;16(1):163.

10. Tucker P, Gilliland J. The effect of season and weather on physical activity: a systematic review. Public Health. 2007;121(12):909-22.

11. Chan CB, Ryan DA. Assessing the effects of weather conditions on physical activity participation using objective measures. Int J Environ Res Public Health. 2009;6(10):2639-54.

12. Baranowski T, Thompson WO, DuRant RH, Baranowski J, Puhl J. Observations on physical activity in physical locations: age, gender, ethnicity, and month effects. Res Q Exerc Sport. 1993;64(2):127-33.

13. Haggarty P, McNeill G, Manneh MK, Davidson L, Milne E, Duncan G, et al. The influence of exercise on the energy requirements of adult males in the UK. Br J Nutr. 1994;72(6):799-813.

14. Rich C, Griffiths $\sqcup$, Dezateux C. Seasonal variation in accelerometerdetermined sedentary behaviour and physical activity in children: a review. Int J Behav Nutr Phys Act. 2012;9(1):49.

15. Chan CB, Ryan DA, Tudor-Locke C. Relationship between objective measures of physical activity and weather: a longitudinal study. Int J Behav Nutr Phys Act. 2006:3:21.

16. The Nobel Peace Prize Award Ceremony 2007 [Internet]. 2007; Oslo, Norway. Available from: NobelPrize.org

17. Friel S, Bowen K, Campbell-Lendrum D, Frumkin H, McMichael AJ, Rasanathan K. Climate change, noncommunicable diseases, and development: the relationships and common policy opportunities. Annu Rev Public Health. 2011;32:133-47.

18. Vogelstein F. The untold story: How the iPhone blew up the wireless industry. Wired [Internet]. 2008; Available from: https://www.wired.com/2 008/01/ff-iphone/

19. About Fitbit [Internet]. Available from: https://www.fitbit.com/us/about-us

20. Ekelund U, Steene-Johannessen J, Brown WJ, Fagerland MW, Owen N, Powell KE, et al. Does physical activity attenuate, or even eliminate, the detrimental association of sitting time with mortality? A harmonised metaanalysis of data from more than 1 million men and women. Lancet. 2016 Sep 24;388(10051):1302-10

21. Stamatakis E, Gale J, Bauman A, Ekelund U, Hamer M, Ding D. Sitting time, physical activity, and risk of mortality in adults. J Am Coll Cardiol. 2019; 73(16):2062-72.

22. Carson V, Spence J. Seasonal variation in physical activity among children and adolescents: a review. Pediatr Exerc Sci. 2010;22:81-92.

23. Munn Z, Peters MDJ, Stern C, Tufanaru C, McArthur A, Aromataris E. Systematic review or scoping review? Guidance for authors when choosing between a systematic or scoping review approach. BMC Med Res Methodol. 2018 Nov 19;18(1):143.

24. U.S. Department of Health and Human Services. 2018 Physical Activity Guidelines Advisory Committee Scientific Report. U.S. Department of Health and Human Services; 2018 p. 779.

25. Ouzzani M, Hammady H, Fedorowicz Z, Elmagarmid A. Rayyan-a web and mobile app for systematic reviews. Systematic Reviews. 2016;5(1):210.

26. Kellermeyer L, Harnke B, Knight S. Covidence and Rayyan. J Med Libr Assoc. 2018:106(4):580-3.

27. Moher D, Liberati A, Tetzlaff J, Altman DG. PRISMA group. Preferred reporting items for systematic reviews and meta-analyses: the PRISMA statement. PLoS Med. 2009;6(7):e1000097-7.
28. Aspvik NP, Viken H, Ingebrigtsen JE, Zisko N, Mehus I, Wisløff U, et al. Do weather changes influence physical activity level among older adults? - the generation 100 study. PLoS One. 2018;13(7):e0199463.

29. Kolle E, Steene-Johannessen J, Andersen LB, Anderssen SA. Seasonal variation in objectively assessed physical activity among children and adolescents in Norway: a cross-sectional study. Int J Behav Nutr Phys Act. 2009 Jun 29;6:36.

30. Koolhaas CM, van Rooij FJA, Schoufour JD, Cepeda M, Tiemeier H, Brage S, et al. Objective measures of activity in the elderly: distribution and associations with demographic and health factors. J Am Med Dir Assoc. 2017 Oct 1;18(10):838-47.

31. Rowlands AV, Pilgrim EL, Eston RG. Seasonal changes in children's physical activity: an examination of group changes, intra-individual variability and consistency in activity pattern across season. Ann Hum Biol. 2009 Jan 1; 36(4):363-78.

32. Shen B, Alexander G, Milberger S, Jen K-LC. An exploratory study of seasonality and preschoolers' physical activity engagement. J Phys Act Health. 2013 Sep;10(7):993-9.

33. Atkin AJ, Sharp SJ, Harrison F, Brage S, Van Sluijs EMF. Seasonal variation in children's physical activity and sedentary time. Med Sci Sports Exerc. 2016 Mar:48(3):449-56.

34. Cepeda M, Koolhaas CM, van Rooij FJA, Tiemeier H, Guxens M, Franco OH et al. Seasonality of physical activity, sedentary behavior, and sleep in a middle-aged and elderly population: the Rotterdam study. Maturitas. 2018 Apr; 110:41-50.

35. Larouche R, Blanchette S, Faulkner G, Riazi N, Trudeau F, Tremblay MS. Correlates of children's physical activity: a Canadian multisite study. Med Sci Sports Exerc. 2019 Dec;51(12):2482-90.

36. Albrecht BM, Stalling I, Recke C, Bammann K. Accelerometer-assessed outdoor physical activity is associated with meteorological conditions among older adults: cross-sectional results from the OUTDOOR ACTIVE study. PLoS One. 2020;15(1):e0228053.

37. Rahman S, Maximova K, Carson V, Jhangri GS, Veugelers PJ. Stay in or play out? The influence of weather conditions on physical activity of grade 5 children in Canada. Can J Public Health. 2019;110(2):169-77.

38. Wu Y-T, Luben R, Wareham N, Griffin S, Jones AP. Weather, day length and physical activity in older adults: cross-sectional results from the European prospective investigation into Cancer and nutrition (EPIC) Norfolk cohort. PLoS One. 2017;12(5):e0177767.

39. Lewis LK, Maher C, Belanger K, Tremblay M, Chaput J-P, Olds T. At the mercy of the gods: associations between weather, physical activity, and sedentary time in children. Pediatr Exerc Sci. 2016;28(1):152-63.

40. Ogawa S, Seko T, Ito T, Mori M. Differences in physical activity between seasons with and without snowfall among elderly individuals residing in areas that receive snowfall. J Phys Ther Sci. 2019 Jan;31(1):12-6.

41. Pagels P, Raustorp A, Guban P, Fröberg A, Boldemann C. Compulsory school in- and outdoors -implications for school children's physical activity and health during one academic year. Int J Environ Res Public Health. 2016;13(7): 699.

42. Patnode CD, Lytle LA, Erickson DJ, Sirard JR, Barr-Anderson D, Story M. The relative influence of demographic, individual, social, and environmental factors on physical activity among boys and girls. Int J Behav Nutr Phys Act. 2010 Nov 3:7(1):79.

43. Duncan JS, Hopkins WG, Schofield G, Duncan EK. Effects of weather on pedometer-determined physical activity in children. Med Sci Sports Exerc. 2008 Aug;40(8):1432-8

44. McCrorie PRW, Duncan E, Granat MH, Stansfield BW. Seasonal variation in the distribution of daily stepping in 11-13 year old school children. Int J Exerc Sci. 2015;8:4

45. World Bank Country and Lending Groups [Internet]. The World Bank. 2020 [cited 2020 Jun 5]. Available from: https://datahelpdesk.worldbank.org/ knowledgebase/articles/906519-world-bank-country-and-lending-groups

46. Ma BD, Ng SL, Schwanen T, Zacharias J, Zhou M, Kawachi I, et al. Pokémon GO and physical activity in Asia: multilevel study. J Med Internet Res. 2018; 20(6):e217.

47. Arnardottir NY, Oskarsdottir ND, Brychta RJ, Koster A, Van Domelen DR, Caserotti P, et al. Comparison of summer and winter objectively measured physical activity and sedentary behavior in older adults: age, gene/ environment susceptibility Reykjavik study. Int J Environ Res Public Health. 2017:14(10):1268. 
48. Edwards NM, Myer GD, Kalkwarf HJ, Woo JG, Khoury PR, Hewett TE, et al. Outdoor temperature, precipitation, and wind speed affect physical activity levels in children: a longitudinal cohort study. J Phys Act Health. 2015 Aug; 12(8):1074-81.

49. Hjorth MF, Chaput J-P, Michaelsen K, Astrup A, Tetens I, Sjödin A. Seasonal variation in objectively measured physical activity, sedentary time, cardio-respiratory fitness and sleep duration among 8-11 year-old Danish children: a repeated-measures study. BMC Public Health. 2013 Sep 8:13:808.

50. Schepps MA, Shiroma EJ, Kamada M, Harris TB, Lee I-M. Day length is associated with physical activity and sedentary behavior among older women. Sci Rep. 2018;8(1):6602

51. Silva P, Santos R, Welk G, Mota J. Seasonal differences in physical activity and sedentary patterns: the relevance of the PA context. J Sports Sci Med. 2011;10(1):66-72.

52. Chang Z, Wang S, Zhang X. Seasonal variations in physical activity and sedentary behavior among preschool children in a Central China city. Am J Hum Biol. 2020 Feb;25:e23406.

53. Nagy L, Faisal M, Horne M, Collings P, Barber S, Mohammed M. Factors associated with accelerometer measured movement behaviours among White British and south Asian children aged 6-8 years during school terms and school holidays. BMJ Open. 2019;9:e025071.

54. Aadland E, Andersen LB, Anderssen SA, Resaland GK, Resaland GK. A comparison of 10 accelerometer non-wear time criteria and logbooks in children. BMC Public Health. 2018;18:1-1.

55. Aebi NJ, Bringolf-Isler B, Schaffner E, Caviezel S, Imboden M, Probst-Hensch N. Patterns of cross-sectional and predictive physical activity in Swiss adults aged 52+: results from the SAPALDIA cohort. Swiss Med Wkly. 2020;150: w20266.

56. Van Kann DHH, de Vries SI, Schipperijn J, de Vries NK, Jansen MWJ, Kremers SPJ. Schoolyard characteristics, physical activity, and sedentary behavior: combining GPS and accelerometry. J Sch Health. 2016;86(12):913-21.

57. Yildirim M, Schoeni A, Singh AS, Altenburg TM, Brug J, De Bourdeaudhuij I, et al. Daily variations in weather and the relationship with physical activity and sedentary time in European 10- to 12-year-olds: the ENERGY-project. J Phys Act Health. 2014 Feb;11(2):419-25.

58. Sit CHP, Huang WY, Yu JJ, McKenzie TL. Accelerometer-assessed physical activity and sedentary time at school for children with disabilities: seasonal variation. Int J Environ Res Public Health. 2019;16(17):3163.

59. Harrison F, Jones AP, Bentham G, van Sluijs EMF, Cassidy A, Griffin SJ. The impact of rainfall and school break time policies on physical activity in 9-10 year old British children: a repeated measures study. Int J Behav Nutr Phys Act. 2011 May 24:8:47

60. Katapally TR, Rainham D, Muhajarine N. The influence of weather variation, urban design and built environment on objectively measured sedentary behaviour in children. AIMS Public Health. 2016;3(4):663-81

61. Harrison F, van Sluijs EMF, Corder K, Ekelund U, Jones A. The changing relationship between rainfall and children's physical activity in spring and summer: a longitudinal study. International Journal of Behavioral Nutrition \& Physical Activity. 2015;12:1-9.

62. Aibar Solana A, Bois JE, Zaragoza J, Bru N, Paillard T, Generelo E. Adolescents' sedentary behaviors in two European cities. Research Quarterly for Exercise \& Sport. 2015;86(3):233-43.

63. Pechová J, Pelclová J, Dygryn J, Zajac-Gawlak I, Tlucakova L. Sedentary behaviour patterns and spring-autumn seasonality in older central European adults. Journal of Physical Education \& Sport. 2019;19(2):1092-8.

64. Davis MG, Fox KR, Hillsdon M, Sharp DJ, Coulson JC, Thompson JL. Objectively measured physical activity in a diverse sample of older urban UK adults. Med Sci Sports Exerc. 2011:43(4):647-54.

65. Hagströmer M, Rizzo NS, Sjöström M. Associations of season and region on objectively assessed physical activity and sedentary behaviour. J Sports Sci. 2014;32(7):629-34.

66. Nilsen AKO, Anderssen SA, Ylvisaaker E, Johannessen K, Aadland E. Physical activity among Norwegian preschoolers varies by sex, age, and season. Scand J Med Sci Sports. 2019;29(6):862-73.

67. O'Connell SE, Griffiths PL, Clemes SA. Seasonal variation in physical activity, sedentary behaviour and sleep in a sample of UK adults. Ann Hum Biol. 2014;41(1):1-8

68. Gracia-Marco L, Ortega FB, Ruiz JR, Williams CA, HagstrÖmer M, Manios Y, et al. Seasonal variation in physical activity and sedentary time in different European regions. The HELENA study. J Sports Sci. 2013;31(16):1831-40.
69. Diaz KM, Howard VJ, Hutto B, Colabianchi N, Vena JE, Blair SN, et al. Patterns of sedentary behavior in US middle-age and older adults: the REGARDS study. Med Sci Sports Exerc. 2016;48(3):430-8.

70. Hunter S, Rosu A, Hesketh KD, Rhodes RE, Rinaldi CM, Rodgers W, et al. Objectively measured environmental correlates of toddlers' physical activity and sedentary behavior. Pediatr Exerc Sci. 2019;31(4):480-7.

71. Wong S, Cantoral A, Téllez-Rojo MM, Pantic I, Oken E, Svensson K, et al. Associations between daily ambient temperature and sedentary time among children 4-6 years old in Mexico City. PLoS One [Internet]. 2020 Oct 30 [cited 2020 Dec 3];15(10). Available from: https://www.ncbi.nlm.nih.gov/ pmc/articles/PMC7598506/

72. Dias Kl, White J, Jago R, Cardon G, Davey R, Janz KF, et al. International comparison of the levels and potential correlates of objectively measured sedentary time and physical activity among three-to-four-year-old children. Int J Environ Res Public Health. 2019;16:11.

73. Kharlova I, Deng WH, Mamen J, Mamen A, Fredriksen MV, Fredriksen PM. The weather impact on physical activity of 6-12 year old children: a clustered study of the Health Oriented Pedagogical Project (HOPP). Sports (Basel). 2020:8:1.

74. Collings PJ, Dogra SA, Costa S, Bingham DD, Barber SE. Objectivelymeasured sedentary time and physical activity in a bi-ethnic sample of young children: variation by socio-demographic, temporal and perinatal factors. BMC Public Health. 2020 Jan 28;20(1):109.

75. King AC, Parkinson KN, Adamson AJ, Murray L, Besson H, Reilly JJ, et al. Correlates of objectively measured physical activity and sedentary behaviour in English children. Eur J Pub Health. 2011 Aug;21(4):424-31.

76. Pearce MS, Basterfield L, Mann KD, Parkinson KN, Adamson AJ, Reilly JJ, et al. Early predictors of objectively measured physical activity and sedentary behaviour in 8-10 year old children: the Gateshead millennium study. PLoS One. 2012;7(6):e37975.

77. Buchowski MS, Choi L, Majchrzak KM, Acra S, Mathews CE, Chen KY Seasonal changes in amount and patterns of physical activity in women. J Phys Act Health. 2009;6(2):252-61

78. Sartini C, Morris RW, Whincup PH, Wannamethee SG, Ash S, Lennon L, et al. Association of maximum temperature with sedentary time in older British men. J Phys Act Health. 2017;14(4):265-9.

79. Remmers T, Thijs C, Timperio A, Salmon J, Veitch J, Kremerr SPJ, et al. Daily weather and children's physical activity patterns. Med Sci Sports Exerc. 2017; 49(5):922-9.

80. Hoaas H, Zanaboni P, Hjalmarsen A, Morseth B, Dinesen B, Burge AT, et al. Seasonal variations in objectively assessed physical activity among people with COPD in two Nordic countries and Australia: a cross-sectional study. Int J Chron Obstruct Pulmon Dis. 2019:14:1219-28.

81. Zheng C, Huang WY, Wong SH-S. Associations of weather conditions with adolescents' daily physical activity, sedentary time, and sleep duration. Applied Physiology, Nutrition \& Metabolism. 2019;44(12):1339-44.

82. Cullen KW, Liu Y, Thompson D. Diet and physical activity in african-american girls: seasonal differences. Am J Health Behav. 2017 Mar 1;41(2):171-8.

83. Bringolf-Isler B, Grize L, Mäder U, Ruch N, Sennhauser FH, Braun-Fahrländer C. Assessment of intensity, prevalence and duration of everyday activities in Swiss school children: a cross-sectional analysis of accelerometer and diary data. Intern J Behav Nutr Phys Act. 2009;6:10.

84. Newman MA, Pettee KK, Storti KL, Richardson CR, Kuller LH, Kriska AM. Monthly variation in physical activity levels in postmenopausal women. Med Sci Sports Exerc. 2009 Feb;41(2):322-7.

85. Barkley SA, Herrmann SD. Seasonal variation of physical Activity in community-living vs. residential-dwelling older adults. Californian Journal of Health Promotion. 2017 Dec;15(3):37-47.

86. Brychta RJ, Arnardottir NY, Johannsson E, Wright EC, Eiriksdottir G, Gudnason V, et al. Influence of day length and physical activity on sleep patterns in older Icelandic men and women. J Clin Sleep Med. 2016;12(2): 203-13.

87. Carr $\sqcup$, Dunsinger S, Marcus BH. Long-term surveillance of physical activity habits of latinas enrolled in a 12-month physical activity intervention. J Phys Act Health. 2016;13(7):740-6.

88. Sumukadas D, Witham M, Struthers A, McMurdo M. Day length and weather conditions profoundly affect physical activity levels in older functionally impaired people. J Epidemiol Community Health. 2009 Apr 1;63(4):305.

89. Nakashima D, Kimura D, Watanabe H, Goto F, Kato M, Fujii K, et al. Influence of seasonal variations on physical activity in older people living in mountainous agricultural areas. J Rural Med. 2019 Nov;14(2):165-75. 
90. Pelclová J, Walid EA, Vašícková J. Study of day, month and season pedometer-determined variability of physical activity of high school pupils in the Czech Republic. J Sports Sci Med. 2010;9(3):490-8.

91. Beighle A, Alderman B, Morgan CF, Le Masurier G. Seasonality in children's pedometer-measured physical activity levels. Res Q Exerc Sport. 2008 Jun; 79(2):256-60.

92. Akande VO, Ruiter RAC, Kremers SPJ. Environmental and motivational determinants of physical activity among Canadian inuit in the arctic. Int J Environ Res Public Health. 2019;09:16(13).

93. Clemes SA, Hamilton SL, Griffiths PL. Summer to winter variability in the step counts of normal weight and overweight adults living in the UK. J Phys Act Health. 2011 Jan 1;8(1):36-44.

94. Cooper AR, Page AS, Wheeler BW, Hillsdon M, Griew P, Jago R. Patterns of GPS measured time outdoors after school and objective physical activity in English children: the PEACH project. Int J Behav Nutr Phys Act. 2010 Dec; 7(1):1-9.

95. Hamilton SL, Clemes SA, Griffiths PL. UK adults exhibit higher step counts in summer compared to winter months. Ann Hum Biol. 2008 Apr;35(2):154-69.

96. Kimura T, Kobayashi H, Nakayama E, Kakihana W. Seasonality in physical activity and walking of healthy older adults. J Physiol Anthropol [Internet]. 2015 Oct 2 [cited 2020 Mar 4];34. Available from: https://www.ncbi.nlm.nih. gov/pmc/articles/PMC4591564/

97. Mitsui T, Barajima T, Kanachi M, Shimaoka K. Daily walking activity among male office workers in a rural town in northern Japan. J Physiol Anthropol. 2010;29(1):43-6

98. Sewell L, Singh SJ, Williams JE, Morgan MD. Seasonal variations affect physical activity and pulmonary rehabilitation outcomes. J Cardiopulm Rehabil Prev. 2010 Oct;30(5):329-33.

99. Hopkins ND, Stratton G, Tinken TM, Ridgers ND, Graves LE, McWhannell N, et al. Seasonal reduction in physical activity and flow-mediated dilation in children. Med Sci Sports Exerc. 2011;43(2):232-8.

100. Ridgers ND, Salmon J, Timperio A. Seasonal changes in physical activity during school recess and lunchtime among Australian children. J Sports Sci. 2018;36(13):1508-14

101. Ridgers ND, Salmon J, Timperio A. Too hot to move? Objectively assessed seasonal changes in Australian children's physical activity. Int J Behav Nutr Phys Act. 2015 Jun 19;12(1):77.

102. Deng WH, Fredriksen PM. Objectively assessed moderate-to-vigorous physical activity levels among primary school children in Norway: The Health Oriented Pedagogical Project (HOPP). Scand J Public Health. 2018; 46(21_suppl):38-47.

103. Bremer E, Graham JD, Bedard C, Rodriguez C, Kriellaars D, Cairney J. The association between PLAYfun and physical activity: a convergent validation study. Res Q Exerc Sport. 2019;16:1-9.

104. Crowley O, Pugliese L, Kachnowski S. The impact of wearable device enabled health initiatives on physical activity and sleep. Cureus. 2016;8(10):e825.

105. Kong S, Park HY, Kang D, Lee JK, Lee G, Kwon OJ, et al. Seasonal variation in physical activity among preoperative patients with lung cancer determined using a wearable device. J Clin Med. 2020;27:9(2).

106. McKee DP, Murtagh EM, Boreham CAG, Nevill AM, Murphy MH. Seasonal and annual variation in young children's physical activity. Med Sci Sports Exerc. 2012;44(7):1318-24.

107. Jones GR, Brandon C, Gill DP. Physical activity levels of community-dwelling older adults are influenced by winter weather variables. Arch Gerontol Geriatr. 2017:71:28-33.

108. de Vries PR, Janssen M, Spaans E, de Groot I, Janssen A, Smeitink J, et al. Natural variability of daily physical activity measured by accelerometry in children with a mitochondrial disease. Mitochondrion. 2019;47:30-7.

109. Harrison F, Goodman A, van Sluijs EMF, Andersen LB, Cardon G, Davey R, et al. Weather and children's physical activity; how and why do relationships vary between countries? Int J Behav Nutr Phys Act. 2017;14(1):74.

110. Robbins SM, Jones GR, Birmingham TB, Maly MR. Quantity and quality of physical activity are influenced by outdoor temperature in people with knee osteoarthritis. Physiother Can. 2013;65(3):248-54.

111. Balish SM, Dechman G, Hernandez P, Spence JC, Rhodes RE, McGannon K, et al. The relationship between weather and objectively measured physical activity among individuals with COPD. J Cardiopulm Rehabil Prev. 2017; 37(6):445-9.

112. Stabell AC, Wilson M, Jankowski CM, MaWhinney S, Erlandson KM. The impact of a structured, supervised exercise program on daily step count in sedentary older adults with and without HIV. JAIDS. 2020;84(2):228-33.
113. Prins RG, van Lenthe FJ. The hour-to-hour influence of weather conditions on walking and cycling among Dutch older adults. Age Ageing. 2015;44(5): 886-90.

114. Witham MD, Donnan PT, Vadiveloo T, Sniehotta FF, Crombie IK, Feng Z et al. Association of day length and weather conditions with physical activity levels in older community dwelling people. PLoS One. 2014;9(1): e85331.

115. Yıldırım M, Schoeni A, Singh AS, Altenburg TM, Brug J, De Bourdeaudhuij I, et al. Daily variations in weather and the relationship with physical activity and sedentary time in European 10- to 12-year-Olds: the ENERGY-project. J Phys Act Health. 2014;11(2):419-25.

116. Button BLG, Shah TI, Clark AF, Wilk P, Gilliland JA. Examining weather-related factors on physical activity levels of children from rural communities. Can J Public Health. 2020.

117. Brandon CA, Gill DP, Speechley M, Gilliland J, Jones GR. Physical activity levels of older community-dwelling adults are influenced by summer weather variables. Applied Physiology, Nutrition \& Metabolism. 2009;34(2):182-90.

118. Badland HM, Christian H, Giles-Corti B, Knuiman M. Seasonality in physical activity: should this be a concern in all settings? Health \& Place. 2011;17(5): 1084-9.

119. Delclòs-Alió X, Marquet O, Vich G, Schipperijn J, Zhang K, Maciejewska M, et al. Temperature and rain moderate the effect of neighborhood walkability on walking time for seniors in Barcelona. Int J Environ Res Public Health. 2019;18:17(1).

120. Wang G, Li B, Zhang X, Niu C, Li J, Li L, et al. No seasonal variation in physical activity of Han Chinese living in Beijing. International Journal of Behavioral Nutrition \& Physical Activity. 2017;14:1-10.

121. Hoppmann CA. Chak man Lee J, Ziegelmann JP, Graf P, khan KM, Ashe MC. Precipitation and physical Activity in older adults: the moderating role of functional mobility and physical activity intentions. J Gerontol Series B: Psychol Sci Soc Sci. 2017;72(5):792-800.

122. Boutou AK, Raste $Y$, Demeyer H, Troosters T, Polkey MI, Vogiatzis I, et al. Progression of physical inactivity in COPD patients: the effect of time and climate conditions - a multicenter prospective cohort study. Int J Chron Obstruct Pulmon Dis. 2019 Sep 3;14:1979-92.

123. Cradock AL, Melly SJ, Allen JG, Morris JS, Gortmaker SL. Youth destinations associated with objective measures of physical activity in adolescents. J Adolesc Health. 2009 Sep 2:45(3):S91-8.

124. Martins RC, Reichert FF, Bielemann RM, Hallal PC. One-year stability of objectively measured physical Activity in young Brazilian adults. J Phys Act Health. 2017;14(3):208-12.

125. Feinglass J, Lee J, Dunlop D, Song J, Semanik P, Chang RW. The effects of daily weather on accelerometer-measured physical activity among adults with arthritis. J Phys Act Health. 2011;8(7):934-43.

126. Jehn M, Gebhardt A, Liebers U, Kiran B, Scherer D, Endlicher W, et al. Heat stress is associated with reduced health status in pulmonary arterial hypertension: a prospective study cohort. Lung. 2014;192(4):619-24.

127. Al-Mohannadi AS, Faroog A, Burnett A, Van Der Walt M, Al-Kuwari MG. Impact of climatic conditions on physical Activity: a 2-year cohort study in the Arabian gulf region. J Phys Act Health. 2016;13(9):929-37.

128. Mitchell DC, Armitage TL, Bennett DH, Schenker MB, Castro J, Tancredi DJ. Physical activity and common tasks of California farm workers: California heat illness prevention study (CHIPS). J Occupational \& Environmental Hygiene. 2018;15(12):857-69.

129. Bejarano CM, Cushing CC, Crick CJ. Does context predict psychological states and activity? An ecological momentary assessment pilot study of adolescents. Psychology of Sport \& Exercise. 2019 Mar;41:146-52.

130. Colom A, Ruiz M, Wärnberg J, Compa M, Muncunill J, Barón-López FJ, et al. Mediterranean built environment and precipitation as modulator factors on physical activity in obese mid-sge and old-age adults with metabolic syndrome: cross-sectional study. Int J Environ Res Public Health. 2019;16(5):854

131. Dill J, McNeil N, Broach J, Ma L. Bicycle boulevards and changes in physical activity and active transportation: findings from a natural experiment. Prev Med. 2014;69(Suppl 1):S74-8.

132. Sugino A, Minakata Y, Kanda M, Akamatsu K, Koarai A, Hirano T, et al. Validation of a compact motion sensor for the measurement of physical activity in patients with chronic obstructive pulmonary disease. Respiration. 2012:83(4):300-7.

133. Oliver M, Schluter PJ, Schofield GM, Paterson J. Factors related to accelerometer-derived physical activity in Pacific children aged 6 years. Asia Pac J Public Health. 2011 Jan;23(1):44-56. 
134. Goodman A, Page AS, Cooper AR. International Children's Accelerometry Database (ICAD) Collaborators. Daylight saving time as a potential public health intervention: an observational study of evening daylight and objectively-measured physical activity among 23,000 children from 9 countries. Int J Behav Nutr Phys Act. 2014;11:84.

135. Goodman A, Paskins J, Mackett R. Day length and weather effects on children's physical Activity and participation in play, sports, and active travel. J Phys Act Health. 2012;9(8):1105-16.

136. Griew P, Page A, Thomas S, Hillsdon M, Cooper AR. The school effect on children's school time physical activity: the PEACH project. Prev Med. 2010; 51(3-4):282-6.

137. McMurdo MET, Argo I, Crombie IK, Feng Z, Sniehotta FF, Vadiveloo T, et al. Social, environmental and psychological factors associated with objective physical activity levels in the over 65s. PLoS One. 2012;7(2):e31878.

138. Rosenthal DG, Vittinghoff E, Tison GH, Pletcher MJ, Olgin JE, Grandis DJ, et al. Assessment of accelerometer-based physical activity during the 20172018 California wildfire seasons. JAMA Netw Open. 2020;3(10):e2018116-6.

139. Institute of Medicine (US). Human health and the natural environment [Internet]. Rebuilding the Unity of Health and the Environment: A New Vision of Environmental Health for the 21st Century. National Academies Press (US); 2001. Available from: http://www.ncbi.nlm.nih.gov/books/NBK995 84/.

140. Taylor SE, Repetti RL, Seeman T. Health psychology: what is an unhealthy environment and how does it get under the skin? Annu Rev Psychol. 1997; 48:411-47.

141. Barreto P. de S. Why are we failing to promote physical activity globally? Bull World Health Organ. 2013;91(6):390-390A.

142. Whitfield G, Carlson S, Ussery E, Fulton J, Galuska D, Petersen R. Trends in meeting physical activity guidelines among urban and rural dwelling adults - United States, 2008-2017. MMWR Morb Mortal Wkly Rep. 2019;68:513-8.

143. Martin KR, Koster A, Murphy RA, Van Domelen DR, Hung M, Brychta RJ, et al. Changes in daily activity patterns with age in U.S. men and women: National Health and nutrition examination survey (NHANES) 2003-04 and 2005-06. J Am Geriatr Soc. 2014 Jul 1;62(7):1263-71.

144. Amagasa S, Machida M, Fukushima N, Kikuchi H, Takamiya T, Odagiri Y, et al. Is objectively measured light-intensity physical activity associated with health outcomes after adjustment for moderate-to-vigorous physical activity in adults? A systematic review. Int J Behav Nutr Phys Act. 2018 Dec;15(1):1-13.

145. Conroy DE, Lagoa CM, Hekler E, Rivera DE. Engineering person-specific behavioral interventions to promote physical Activity. Exerc Sport Sci Rev. 2020;48(4):170-9.

146. Xu C, Kohler TA, Lenton TM, Svenning J-C, Scheffer M. Future of the human climate niche. Proc Natl Acad Sci U S A. 2020 May;4:201910114.

147. World Health Organization, editor. Global health risks: mortality and burden of disease attributable to selected major risks. Geneva, Switzerland: World Health Organization; 2009. 62 p.

148. Population estimates and projections [Internet]. DataBank. 2020 [cited 2020 Jun 5]. Available from: https://databank.worldbank.org/source/populationestimates-and-projections

149. Molenaar PCM. On the necessity to use person-specific data analysis approaches in psychology. Eur J Dev Psychol. 2013 Jan 1;10(1):29-39.

150. Oxford English Dictionary (OED) [Internet]. Vol. 2. Oxford, England: Oxford University Press (OUP); 1989. 21,728. Available from: https:/www.oed.com/

151. National Weather Service. National Weather Service Glossary [Internet]. National Oceanic and Atmospheric Administration's National Weather Service. 2009 [cited 2020 Jun 5]. Available from: https://w1.weather.gov/ glossary/

\section{Publisher's Note}

Springer Nature remains neutral with regard to jurisdictional claims in published maps and institutional affiliations.

Ready to submit your research? Choose BMC and benefit from:

- fast, convenient online submission

- thorough peer review by experienced researchers in your field

- rapid publication on acceptance

- support for research data, including large and complex data types

- gold Open Access which fosters wider collaboration and increased citations

- maximum visibility for your research: over $100 \mathrm{M}$ website views per year

At BMC, research is always in progress.

Learn more biomedcentral.com/submissions 